\title{
FINITE-SHEETED COVERING MAPS OVER KLEIN BOTTLE WEAK SOLENOIDAL SPACES
}

\author{
Vlasta Matijević \\ University of Split, Croatia \\ Dedicated to Professor Sibe Mardešić on the occasion of his 80th birthday
}

Abstract. Klein bottle weak solenoidal space $\Sigma(\mathbf{p}, \mathbf{q}, \mathbf{r})$ is a continuum obtained as the inverse limit of an inverse sequence, where each term is Klein bottle and each bonding map is finite-sheeted covering map over Klein bottle. In the present paper we determine and present all $s$-sheeted covering maps (with connected total space) over $\Sigma(\mathbf{p}, \mathbf{q}, \mathbf{r}), s \in \mathbb{N}$, both pointed and unpointed case.

\section{INTRODUCTION AND THE MAIN RESULT}

Recently, finite-sheeted covering maps over 2-dimensional compact, connected Abelian groups $G$ were studied ([1]). It turned out that finite-sheeted covering maps over $G$ were determined using finite-index torsion free supergroups of the Pontryagin dual $\widehat{G}([2])$. Moreover, using finite index subgroups of $\widehat{G}$ there were also presented finite-sheeted covering maps from $G$ to other compact connected groups. The main step in the investigation was the reduction to the case of finite-sheeted covering homomorphisms $f: G^{\prime} \rightarrow G$ between two compact connected 2-dimensional Abelian groups. Each such group $G$ is represented as the inverse limit of an inverse sequence, where each term is 2 -torus $\mathbb{T}^{2}$ and each bonding map is a finite-sheeted covering homomorphism over $\mathbb{T}^{2}$. Since $\mathbb{T}^{2}$ is a covering space for Klein bottle $K$, a natural

2000 Mathematics Subject Classification. 14H30, 22C05, 57M10.

Key words and phrases. Inverse system, covering map, Klein bottle, weak solenoidal space, Klein bottle weak solenoidal space, 2-dimensional torus, 2-dimensional compact Abelian group, fundamental progroup, subprogroup of index $s$, conjugacy classes of subprogroups of index $s$. 
question arises: Are compact connected Abelian 2-dimensional groups $G$, besides groups, also covering spaces for 2-dimensional continua $Y$ obtained as limits of inverse sequences consisting of $K$ ? This question leads us to an investigation of finite-sheeted covering maps over Klein bottle weak solenoidal continua $\Sigma(\boldsymbol{p}, \boldsymbol{q}, \boldsymbol{r})$, where $\boldsymbol{p}=\left(p_{i}\right), \boldsymbol{q}=\left(q_{i}\right)$ and $\boldsymbol{r}=\left(r_{i}\right)$ are sequences of integers such that $p_{i} \neq 0$ and $r_{i}$ is odd for each $i$. Weak solenoidal spaces $\Sigma(\boldsymbol{p}, \boldsymbol{q}, \boldsymbol{r})$ were introduced and classified up to homeomorphism by C. Tezer in his paper "Shape classification of Klein bottle-like continua" ([7]). The aim of the present paper is to determine and present all $s$-sheeted covering maps with connected total space over $\Sigma(\boldsymbol{p}, \boldsymbol{q}, \boldsymbol{r}), s \in \mathbb{N}$, both pointed and unpointed case. Main results related to the pointed case are given in Theorem 6.2 and Corollary 6.6, while main results related to the unpointed case are given in Theorem 7.3 and Corollary 7.5. The results are achieved using classification theorem of finite-sheeted covering maps over connected paracompact spaces $Y([5])$. It establishes a bijection between the set of all pointed equivalence classes of $s$-sheeted pointed covering maps $f:(X, *) \rightarrow(Y, *)$ and the set of all subprogroups of index $s$ of the fundamental progroup $\pi_{1}(Y, *)$. In the unpointed case it establishes a bijection between the set of all equivalence classes of $s$-sheeted maps $f: X \rightarrow Y$ and the set of all conjugacy classes of subprogroups of index $s$ of the fundamental progroup $\pi_{1}(Y, *)$, where $*$ is an arbitrary chosen point of $Y$. It turned out that the investigation of $s$-sheeted covering maps over $\Sigma(\boldsymbol{p}, \boldsymbol{q}, \boldsymbol{r})$ was reduced to the studying of certain sequences of positive integers, so called admissible sequences for $\Sigma(\boldsymbol{p}, \boldsymbol{q}, \boldsymbol{r})$, and their conjugacy classes.

\section{SPACES $\Sigma(\boldsymbol{p}, \boldsymbol{q}, \boldsymbol{r})$}

We shall follow notions introduced by Tezer in [7]. Klein bottle $K$ can be presented as the quotient manifold $\mathbb{R}^{2} / G$, where the group $G=\langle\alpha, \beta|$ $\left.\alpha \beta=\beta \alpha^{-1}\right\rangle$ acts properly discontinuously on $\mathbb{R}^{2}$ by the affine transformations $\alpha, \beta: \mathbb{R}^{2} \rightarrow \mathbb{R}^{2}$

$$
\begin{aligned}
& \alpha(x, y)=(x+1, y) \\
& \beta(x, y)=\left(-x, y+\frac{1}{2}\right)
\end{aligned}
$$

Let $y_{0} \in K$ be the image of $(0,0) \in \mathbb{R}^{2}$ under the quotient map. Then $\pi_{1}\left(K, y_{0}\right)$ can be naturally identified with $G$. Each element of $G$ can be presented as $\alpha^{n} \beta^{m}, n, m \in \mathbb{Z}$. Note that $G$ can be viewed as the group $\left(\mathbb{Z}^{2}, *\right)$, where the group operation $*$ is given by $(n, m) *(k, l)=\left(n+(-1)^{m} k, m+l\right)$. Namely, $h: G \rightarrow\left(\mathbb{Z}^{2}, *\right)$ defined by the rule $h\left(\alpha^{n} \beta^{m}\right)=(n, m)$ is an isomorphism of groups. Therefore we shall identify $G$ with $\left(\mathbb{Z}^{2}, *\right)$ via $h$. In the 
sequel we shall need following relations:

$$
\begin{aligned}
& \left(\alpha^{n} \beta^{m}\right)^{k}= \begin{cases}\alpha^{k n} \beta^{k m}, & m \text { even } \\
\beta^{k m}, & m \text { odd, } k \text { even }, k \in \mathbb{Z} \backslash\{0\}, \\
\alpha^{n} \beta^{k m}, & m \text { odd, } k \text { odd }\end{cases} \\
& \left(\alpha^{n} \beta^{m}\right)^{-1}=\alpha^{(-1)^{m+1} n} \beta^{-m} .
\end{aligned}
$$

Also note that the subgroup $(\mathbb{Z} \times 2 \mathbb{Z}, *)$ of $\left(\mathbb{Z}^{2}, *\right)$ is isomorphic to $\left(\mathbb{Z}^{2},+\right)$. Since $G^{\prime}=\left\langle\alpha, \beta^{2}\right\rangle=(\mathbb{Z} \times 2 \mathbb{Z}, *)$, it follows that the the quotient manifold $\mathbb{R}^{2} / G^{\prime}$ is the 2-torus $\mathbb{T}^{2}$. Let $x_{0} \in \mathbb{T}^{2}$ be the image of $(0,0) \in \mathbb{R}^{2}$ under the quotient map. Then $\pi_{1}\left(\mathbb{T}^{2}, x_{0}\right)=G^{\prime}$. Since $G^{\prime}$ is a subgroup of index 2 of $G$, the identity map $i d_{\mathbb{R}^{2}}: \mathbb{R}^{2} \rightarrow \mathbb{R}^{2}$ induces a pointed map $\delta:\left(\mathbb{T}^{2}, x_{0}\right) \rightarrow\left(K, y_{0}\right)$, which is a 2 -sheeted covering map, so called "basic" 2-sheeted covering map of $\mathbb{T}^{2}$ over $K$.

Each endomorphism of $G$ is of the form $h_{(p, q, r)}: G \rightarrow G$

$$
\begin{aligned}
& h_{(p, q, r)}(\alpha)=\alpha^{p}, \\
& h_{(p, q, r)}(\beta)=\alpha^{q} \beta^{r}
\end{aligned}
$$

where $p, q, r \in \mathbb{Z}$ and $r$ is odd whenever $p \neq 0$. Moreover, $h_{(p, q, r)}$ is injective if and only if $p \neq 0$ (and $r$ is odd). Furthermore,

$$
h_{(p, q, r)} h_{\left(p^{\prime}, q^{\prime}, r^{\prime}\right)}=h_{\left(p p^{\prime}, t, r r^{\prime}\right)}, \text { where } \begin{cases}t=q r^{\prime}, & r \text { even } \\ t=p q^{\prime}, & r \text { odd, } r^{\prime} \text { even . } \\ t=p q^{\prime}+q, & r \text { odd, } r^{\prime} \text { odd }\end{cases}
$$

For each integers $p, q$ and $r, r$ odd if $p \neq 0$, Tezer introduced maps $f_{(p, q, r)}$ : $\left(K, y_{0}\right) \rightarrow\left(K, y_{0}\right)$ in the following way. Let $\Theta_{(p, q, r)}:(\mathbb{R}, 0) \rightarrow(\mathbb{R}, 0)$ be a map such that $\Theta_{(p, q, r)}\left(y+\frac{1}{2}\right)=-\Theta_{(p, q, r)}(y)+q$ and define $F_{(p, q, r)}: \mathbb{R}^{2} \rightarrow \mathbb{R}^{2}$ by

$$
F_{(p, q, r)}(x, y)=\left\{\begin{array}{ll}
\left(p x+\Theta_{(p, q, r)}(y), r y\right), & r \text { odd } \\
(2 q y, r y), & r \text { even, } p=0
\end{array} .\right.
$$

$F_{(p, q, r)}$ is the lifting of a map $f_{(p, q, r)}:\left(K, y_{0}\right) \rightarrow\left(K, y_{0}\right)$ such that $f_{(p, q, r) \#}=$ $h_{(p, q, r)} \cdot f_{(p, q, r)}$ is a covering map if and only if $h_{(p, q, r)}$ is injective or equivalently $p \neq 0$. In that case number of sheets equals $|p r|$. Note that if $q=0$ and $p \neq 0, \Theta_{(p, q, r)}$ can be chosen to be the constant function $\Theta_{(p, q, r)}=0$. Then $F_{(p, 0, r)}$ is represented by the diagonal integral matrix $\left[\begin{array}{ll}p & 0 \\ 0 & r\end{array}\right] \in M_{2}(\mathbb{Z})$.

Let $\boldsymbol{p}=\left(p_{i}\right), \boldsymbol{q}=\left(q_{i}\right)$ and $\boldsymbol{r}=\left(r_{i}\right)$ be sequences of integers such that each $p_{i} \neq 0$ and $r_{i}$ is odd. Let $\boldsymbol{Y}=\left\{K_{i}, f_{i i+1}, \mathbb{N}\right\}$ be an inverse sequence such that each $K_{i}=K$ and each bonding map $f_{i i+1}=f_{\left(p_{i}, q_{i}, r_{i}\right)}: K \rightarrow K$ and let $\Sigma(\boldsymbol{p}, \boldsymbol{q}, \boldsymbol{r})$ be the inverse limit of $\boldsymbol{Y} . \Sigma(\boldsymbol{p}, \boldsymbol{q}, \boldsymbol{r})$ is a Klein bottle weak solenoidal space in the sense of McCord (see [6]). Recall the definition.

Definition 2.1. A solenoidal (weak solenoidal) sequence is an inverse sequence $\left\{X_{i}, g_{i i+1}, \mathbb{N}\right\}$ such that each $X_{i}$ is connected, locally pathwise connected and semilocally 1-connected, and each bonding map $g_{i i+1}: X_{i+1} \rightarrow X_{i}$ 
is a regular covering map (covering map). The limit space $\lim _{\longleftarrow}\left\{X_{i}, g_{i i+1}, \mathbb{N}\right\}$ is called a solenoidal (weak solenoidal) space.

Tezer proved that Klein bottle weak solenoidal spaces $\Sigma(\boldsymbol{p}, \boldsymbol{q}, \boldsymbol{r})$ and $\Sigma\left(\boldsymbol{p}^{\prime}, \boldsymbol{q}^{\prime}, \boldsymbol{r}^{\prime}\right)$ are homeomorphic if and only if the sequences $\boldsymbol{p}, \boldsymbol{r}$ and $\boldsymbol{p}^{\prime}, \boldsymbol{r}^{\prime}$ respectively, have essentially the same prime profiles ([7, Proposition 2.5]). In particular, $\Sigma(\boldsymbol{p}, \boldsymbol{q}, \boldsymbol{r})$ and $\Sigma(\boldsymbol{p}, \mathbf{0}, \boldsymbol{r})$ are homeomorphic.

Let $y=\left(y_{i}\right) \in \Sigma(\boldsymbol{p}, \boldsymbol{q}, \boldsymbol{r})$ be a point where each $y_{i}=y_{0} \in K$. When we consider a pointed continuum $(\Sigma(\boldsymbol{p}, \boldsymbol{q}, \boldsymbol{r}), *)$ we will always assume $*=y$ or equivalently $(\Sigma(\boldsymbol{p}, \boldsymbol{q}, \boldsymbol{r}), *)=\lim _{*} \boldsymbol{Y}_{*}=\varliminf_{\mathfrak{l i m}}\left\{\left(K, y_{0}\right), f_{\left(p_{i}, q_{i}, r_{i}\right)}, \mathbb{N}\right\}$.

Recall that each compact connected 2-dimensional Abelian group $A$ is a solenoidal space obtained as the limit of a solenoidal sequence, where each term is 2-torus $\mathbb{T}^{2}$ and each bonding map is a covering homomorphism. That is why we call compact connected 2-dimensional Abelian groups toroidal groups for short.

\section{Subgroups of finite index of $G=\left\langle\alpha, \beta \mid \alpha \beta=\beta \alpha^{-1}\right\rangle$ AND their CONJUGACY CLASSES}

Proposition 3.1. Let $C$ be a cyclic subgroup of $G$. Then the index $[G: C]$ of $C$ in $G$ is infinite.

Proof. Let $C=\left\langle\alpha^{n} \beta^{m}\right\rangle$. If $m$ is even then $C$ is a subgroup of $G^{\prime}=$ $\left\langle\alpha, \beta^{2}\right\rangle$. Since $G^{\prime}$ is isomorphic to $\left(\mathbb{Z}^{2},+\right)$, it follows that $C$ is of infinite index in $G^{\prime}$. Hence $C$ is of infinite index in $G$. Let $m$ be odd. Since $\left(\alpha^{n} \beta^{m}\right)^{2}=\beta^{2 m}$ it follows that $\left\langle\beta^{2 m}\right\rangle \leqslant C$ and $\left[C:\left\langle\beta^{2 m}\right\rangle\right]$ is finite. On the other hand $\left\langle\beta^{2 m}\right\rangle \leqslant G^{\prime}$ and thus the index $\left[G:\left\langle\beta^{2 m}\right\rangle\right]$ is infinite. Now we conclude that $[G: C]$ is infinite.

Proposition 3.2. Let $H$ be an arbitrary subgroup of $G$. Then there is a unique integer $c(H) \in \mathbb{N} \cup\{0\}$ such that pr $r_{2}(H)=c(H) \in \mathbb{Z}$, where $\mathrm{pr}_{2}$ : $G \rightarrow \mathbb{Z}$ is the projection on the second coordinate. If $H$ is non-cyclic then $c(H)>0$.

Proof. First note that $p r_{2}: G \rightarrow \mathbb{Z}$ is a homomorphism of groups. Since $\operatorname{pr}_{2}(H)$ is a subgroup of $\mathbb{Z}$ there is a unique integer $c(H) \in \mathbb{N} \cup\{0\}$, such that $\operatorname{pr}_{2}(H)=c(H) \mathbb{Z}$. Let $H$ be a non-cyclic group and let us assume that $c(H)=0$. Then $H$ is a subgroup of $(\mathbb{Z} \times\{0\}, *)$. The group $(\mathbb{Z} \times\{0\}, *)$ is isomorphic to $(\mathbb{Z} \times\{0\},+)$. Thus $H$ is cyclic, which is a contradiction.

Proposition 3.3. Let $H$ be a non-cyclic subgroup of $G$. If $(0, c(H)) \in H$, then there is a unique $a \in \mathbb{N}$ such that $H=\left\langle\alpha^{a}, \beta^{c(H)}\right\rangle$.

Proof. Since $H$ is non-cyclic, there is an element $(n, m c(H)) \in H, n \in$ $\mathbb{Z} \backslash\{0\}, m \in \mathbb{Z}$. Then $(n, 0) \in H$ and $a=\min \{n \in \mathbb{N}:(n, 0) \in H\}$ is a well-defined natural number. We claim that $H=\left\langle\alpha^{a}, \beta^{c(H)}\right\rangle$. It is obvious 
that $\left\langle\alpha^{a}, \beta^{c(H)}\right\rangle \subseteq H$, so it is enough to prove that $H \subseteq\left\langle\alpha^{a}, \beta^{c(H)}\right\rangle$. Let $(k, l c(H)) \in H, k, l \in \mathbb{Z}$. Then $(k, l c(H))(0, l c(H))^{-1}=(k, 0) \in H$, which implies that $a$ divides $k$. Let $k^{\prime} \in \mathbb{Z}$ be such that $k=k^{\prime} a$. Then $(k, l c(H))=$ $\left(k^{\prime} a, l c(H)\right)=\left(\alpha^{a}\right)^{k^{\prime}}\left(\beta^{c(H)}\right)^{l}$, which shows that $(k, l c(H)) \in\left\langle\alpha^{a}, \beta^{c(H)}\right\rangle$. It remains to prove that $a$ is unique. Let as assume that there is another $a^{\prime} \in \mathbb{N}$ such that $H=\left\langle\alpha^{a^{\prime}}, \beta^{c(H)}\right\rangle$. Then there are $n^{\prime}, n^{\prime \prime} \in \mathbb{N}$ such that $a=n^{\prime} a^{\prime}$ and $a^{\prime}=n^{\prime \prime} a$. This implies $n^{\prime} n^{\prime \prime}=1$, i.e., $a=a^{\prime}$.

Let $k_{0} \in \mathbb{Z}$ be an arbitrary integer and let $f_{k_{0}}: G \rightarrow G$ be a map defined by $f_{k_{0}}(n, m)=\left(n+\frac{1+(-1)^{m+1}}{2} k_{0}, m\right) . f_{k_{0}}$ is an automorphism of the group $G$. Also note that $f_{k_{0}} f_{l_{0}}=f_{k_{0}+l_{0}}$.

Proposition 3.4. Let $H$ be a non-cyclic subgroup of $G$. Then there are unique integers $a, b$ and $c, a, c \in \mathbb{N}, b \in \mathbb{N} \cup\{0\}, 0 \leq b<a$, such that $H=\left\langle\alpha^{a}, \alpha^{b} \beta^{c}\right\rangle$. Furthermore, $[G: H]=a c$.

Proof. Put $c=c(H)$. According to Proposition 3.2, $c>0$. We distinguish two cases.

(i) $c$ is even. If $c$ is even then $H$ is a subgroup of $(\mathbb{Z} \times 2 \mathbb{Z}, *)$, which is isomorphic to $\left(\mathbb{Z}^{2},+\right)$. Thus there are unique numbers $a \in \mathbb{N}$ and $b \in \mathbb{N} \cup\{0\}$, $0 \leq b<a$, such that $H=\left\langle\alpha^{a}, \alpha^{b} \beta^{c}\right\rangle$.

(ii) $c$ is odd. $H$ contains an element $(k, c), k \in \mathbb{Z}$. Let us consider the automorphism $f_{-k}: G \rightarrow G$. Note that $f_{-k}(k, c)=(0, c)$ and $c\left(f_{-k}(H)\right)=$ $c(H)=c$. Now, Proposition 3.3 implies that there is a unique $a \in \mathbb{N}$ such that $f_{-k}(H)=\left\langle\alpha^{a}, \beta^{c}\right\rangle$. Then $H=f_{k} f_{-k}(H)=\left\langle\alpha^{a}, \alpha^{k} \beta^{c}\right\rangle$. Let $b \in \mathbb{N} \cup\{0\}$, $0 \leq b<a$, be such that $k \equiv b(\bmod a)$. Then $H=\left\langle\alpha^{a}, \alpha^{b} \beta^{c}\right\rangle$.

It remains to prove that $[G: H]=a c$. It is enough to prove that

$$
G / H=\left\{H \alpha^{n} \beta^{m}: n, m \in \mathbb{N} \cup\{0\}, 0 \leq n<a, 0 \leq m<c\right\} .
$$

Let us assume $H \alpha^{i} \beta^{j} \cap H \alpha^{n} \beta^{m} \neq \emptyset, 0 \leq i, n<a, 0 \leq j, m<c$. Then $\alpha^{i} \beta^{j}\left(\alpha^{n} \beta^{m}\right)^{-1} \in H$, i.e., $\alpha^{i+(-1)^{j+m+1} n} \beta^{j-m} \in H$, which implies $\alpha^{i+(-1)^{j+m+1} n} \beta^{j-m}=\alpha^{k a}\left(\alpha^{b} \beta^{c}\right)^{l}$ for some $k, l \in \mathbb{Z}$. Since $0 \leq j, m<c$ and $c$ divides $j-m$, it follows $j=m$. Then $\alpha^{i+(-1)^{j+m+1} n}=\alpha^{i-n}=\alpha^{k a}$, which implies $i=n$. Let $\alpha^{k} \beta^{l}, k, l \in \mathbb{Z}$, be an arbitrary element of $G$ and let $l \equiv m(\bmod c), 0 \leq m<c$. Then $l=l_{0} c+m, l_{0} \in \mathbb{Z}$, and $\alpha^{k} \beta^{l}=\alpha^{k} \beta^{l_{0} c+m}$.

Note

$$
\alpha^{k} \beta^{l}= \begin{cases}\alpha^{k-b l_{0}}\left(\alpha^{b} \beta^{c}\right)^{l_{0}} \beta^{m}, & c \text { even } \\ \alpha^{k}\left(\alpha^{b} \beta^{c}\right)^{l_{0}} \beta^{m}, & c \text { odd, } l_{0} \text { even } . \\ \alpha^{k-b}\left(\alpha^{b} \beta^{c}\right)^{l_{0}} \beta^{m}, & c \text { odd, } l_{0} \text { odd }\end{cases}
$$

If $c$ is even put $k-b l_{0} \equiv n(\bmod a), 0 \leq n<a$. If $c$ is odd and $l_{0}$ is even put $k \equiv n(\bmod a), 0 \leq n<a$. If $c$ and $l_{0}$ are odd put $b-k \equiv n(\bmod a)$, $0 \leq n<a$. In all cases $\alpha^{k} \beta^{l} \in H \alpha^{n} \beta^{m}$, which completes the proof.

Proposition 3.1 and Proposition 3.4 imply the following corollary. 
Corollary 3.5. Let $H$ be an arbitrary subgroup of $G$. $[G: H]$ is finite if and only if $H$ is non-cyclic.

Note that all Abelian non-cyclic subgroups of $G$ are contained in the subgroup $G^{\prime}=\left\langle\alpha, \beta^{2}\right\rangle$.

Let $H=\left\langle\alpha^{a}, \alpha^{b} \beta^{c}\right\rangle, a, b \in \mathbb{N}, b \in \mathbb{Z}, 0 \leq b<a$, be a subgroup of $G$. We want to determine a conjugacy class $[H]$ of $H$. First note

$$
\begin{aligned}
& \left(\alpha^{n} \beta^{m}\right)^{-1} \alpha^{a}\left(\alpha^{n} \beta^{m}\right)=\alpha^{(-1)^{m} a}, \\
& \left(\alpha^{n} \beta^{m}\right)^{-1} \alpha^{b} \beta^{2 d}\left(\alpha^{n} \beta^{m}\right)=\alpha^{(-1)^{m} b} \beta^{2 d}, \\
& \left(\alpha^{n} \beta^{m}\right)^{-1} \alpha^{b} \beta^{2 d+1}\left(\alpha^{n} \beta^{m}\right)=\alpha^{(-1)^{m}(b-2 n)} \beta^{2 d+1}, \\
& \left(\alpha^{n} \beta^{m}\right) \alpha^{a}\left(\alpha^{n} \beta^{m}\right)^{-1}=\alpha^{(-1)^{m} a}, \\
& \left(\alpha^{n} \beta^{m}\right) \alpha^{b} \beta^{2 d}\left(\alpha^{n} \beta^{m}\right)^{-1}=\alpha^{(-1)^{m} b} \beta^{2 d}, \\
& \left(\alpha^{n} \beta^{m}\right) \alpha^{b} \beta^{2 d+1}\left(\alpha^{n} \beta^{m}\right)^{-1}=\alpha^{(-1)^{m} b+2 n} \beta^{2 d+1} .
\end{aligned}
$$

Proposition 3.6. Let $a, a^{\prime}, c, c^{\prime} \in \mathbb{N}, 0 \leq b<a, 0 \leq b^{\prime}<a^{\prime}$, and let $H=\left\langle\alpha^{a}, \alpha^{b} \beta^{c}\right\rangle$ and $H^{\prime}=\left\langle\alpha^{a^{\prime}}, \alpha^{b^{\prime}} \beta^{c^{\prime}}\right\rangle$ be conjugate subgroups of $G$. Then $a=a^{\prime}$ and $c=c^{\prime}$.

Proof. Let $g=\alpha^{n} \beta^{m} \in G$ be such that $H^{\prime}=g^{-1} H g$. Since $[G: H]=\left[\begin{array}{ll}G: H^{\prime}\end{array}, a c=a^{\prime} c^{\prime}\right.$. Let $k$ be an integer such that $\alpha^{a^{\prime}}=$ $\left(\alpha^{n} \beta^{m}\right)^{-1} \alpha^{k a}\left(\alpha^{n} \beta^{m}\right)=\alpha^{(-1)^{m} k a}$. It follows that $a$ divides $a^{\prime}$. Analogously, $\alpha^{a}=\left(\alpha^{n} \beta^{m}\right) \alpha^{l a^{\prime}}\left(\alpha^{n} \beta^{m}\right)^{-1}=\alpha^{(-1)^{m} l a^{\prime}}$ for some integer $l$, which shows that $a^{\prime}$ divides $a$. Since $a$ and $a^{\prime}$ are positive it follows $a=a^{\prime}$ and consequently $c=c^{\prime}$.

Proposition 3.7. Let $a, c \in \mathbb{N}, 0 \leq b, b^{\prime}<a$, both $a$ and $c$ odd. Then $H=\left\langle\alpha^{a}, \alpha^{b} \beta^{c}\right\rangle$ and $H^{\prime}=\left\langle\alpha^{a}, \alpha^{b^{\prime}} \beta^{c}\right\rangle$ are conjugate subgroups of $G$.

Proof. Let $n$ be a unique solution of an equation $2 n \equiv b-b^{\prime}(\bmod a)$. Then there is an integer $k$ such that $b-b^{\prime}-2 n=k a$. Put $g=\alpha^{n} \beta^{2}$. We claim $H^{\prime}=g^{-1} H g$. Note that $\alpha^{a}=\left(\alpha^{n} \beta^{2}\right)^{-1} \alpha^{a}\left(\alpha^{n} \beta^{2}\right)$ and $\alpha^{b^{\prime}} \beta^{c}=$ $\alpha^{b-2 n-k a} \beta^{c}=\left(\alpha^{n} \beta^{2}\right)^{-1} \alpha^{b-k a} \beta^{c}\left(\alpha^{n} \beta^{2}\right)$, which shows that $H^{\prime} \subseteq g^{-1} H g$. On the other hand $\alpha^{a}=\left(\alpha^{n} \beta^{2}\right) \alpha^{a}\left(\alpha^{n} \beta^{2}\right)^{-1}$ and $\alpha^{b} \beta^{c}=\alpha^{b^{\prime}+2 n+k a} \beta^{c}=$ $\left(\alpha^{n} \beta^{2}\right) \alpha^{b^{\prime}+k a} \beta^{c}\left(\alpha^{n} \beta^{2}\right)^{-1}$, which shows $H \subseteq g H^{\prime} g^{-1}$.

Proposition 3.8. Let $a, c \in \mathbb{N}, 0 \leq b, b^{\prime}\left\langle a, c\right.$ even. $H=\left\langle\alpha^{a}, \alpha^{b} \beta^{c}\right\rangle$ and $H^{\prime}=\left\langle\alpha^{a}, \alpha^{b^{\prime}} \beta^{c}\right\rangle$ are conjugate subgroups of $G$ if and only if $b^{\prime}=b$ or $b^{\prime}=a-b$.

Proof. Assume that $H$ and $H^{\prime}$ are conjugate subgroups of $G$. Let $g=$ $\alpha^{n} \beta^{m} \in G$ be such that $H^{\prime}=g^{-1} H g$. Then there is an integer $k$ such that

$$
\begin{aligned}
\alpha^{b^{\prime}} \beta^{c} & =\left(\alpha^{n} \beta^{m}\right)^{-1} \alpha^{k a} \alpha^{b} \beta^{c}\left(\alpha^{n} \beta^{m}\right) \\
& =\left(\alpha^{n} \beta^{m}\right)^{-1} \alpha^{k a+b} \beta^{c}\left(\alpha^{n} \beta^{m}\right)=\alpha^{(-1)^{m}(k a+b)} \beta^{c} .
\end{aligned}
$$


Hence $b^{\prime}=(-1)^{m}(k a+b)$, which implies $b^{\prime} \equiv b(\bmod a)$ or $b^{\prime} \equiv-b \equiv a-b$ $(\bmod a)$. This shows $b^{\prime}=b$ or $b^{\prime}=a-b$. Assume $b^{\prime}=b$ or $b^{\prime}=a-b$. If $b=b^{\prime}$ then $H=H^{\prime}$. So let us consider the case $b^{\prime}=a-b$. Put $g=\beta$. Then $\beta^{-1} H \beta=\beta^{-1}\left\langle\alpha^{a}, \alpha^{b} \beta^{c}\right\rangle \beta=\left\langle\alpha^{-a}, \alpha^{-b} \beta^{c}\right\rangle=\left\langle\alpha^{a}, \alpha^{a-b} \beta^{c}\right\rangle=\left\langle\alpha^{a}, \alpha^{b^{\prime}} \beta^{c}\right\rangle=$ $H^{\prime}$.

Proposition 3.9. Let $a, c \in \mathbb{N}, 0 \leq b, b^{\prime}<a$, a even, $c$ odd. $H=$ $\left\langle\alpha^{a}, \alpha^{b} \beta^{c}\right\rangle$ and $H^{\prime}=\left\langle\alpha^{a}, \alpha^{b^{\prime}} \beta^{c}\right\rangle$ are conjugate subgroups of $G$ if and only if $b-b^{\prime}$ is even.

Proof. Assume that $H$ and $H^{\prime}$ are conjugate subgroups of $G$. Let $g=$ $\alpha^{n} \beta^{m} \in G$ be such that $H^{\prime}=g^{-1} H g$. Then there is an integer $k$ such that

$$
\begin{aligned}
\alpha^{b^{\prime}} \beta^{c} & =\left(\alpha^{n} \beta^{m}\right)^{-1} \alpha^{k a} \alpha^{b} \beta^{c}\left(\alpha^{n} \beta^{m}\right) \\
& =\left(\alpha^{n} \beta^{m}\right)^{-1} \alpha^{k a+b} \beta^{c}\left(\alpha^{n} \beta^{m}\right)=\alpha^{(-1)^{m}(k a+b-2 n)} \beta^{c} .
\end{aligned}
$$

It follows $b^{\prime}=k a+b-2 n$ or $b^{\prime}=-k a-b+2 n$. We get $b-b^{\prime} \equiv 2 n(\bmod a)$ or $b+b^{\prime} \equiv 2 n(\bmod a)$. Let $u$ be an integer such that $0 \leq u \leq \frac{a}{2}$ and $2 n \equiv 2 u$ $(\bmod a)$. If $b-b^{\prime} \equiv 2 n(\bmod a)$ we conclude $b-b^{\prime}=2 u$. If $b+b^{\prime} \equiv 2 n(\bmod a)$ then $b-b^{\prime}=b+b^{\prime}-2 b^{\prime}=2 u-2 b^{\prime}=2\left(u-b^{\prime}\right)$. In both cases $b-b^{\prime}$ is even.

Assume that $b-b^{\prime}$ is even. Then there is a solution $n$ of an equation $2 n \equiv b-b^{\prime}(\bmod a)$. Put $g=\alpha^{n} \beta^{2}$. Then $\left(\alpha^{n} \beta^{2}\right)^{-1} H\left(\alpha^{n} \beta^{2}\right)=$ $\left(\alpha^{n} \beta^{2}\right)^{-1}\left\langle\alpha^{a}, \alpha^{b} \beta^{c}\right\rangle\left(\alpha^{n} \beta^{2}\right)=\left\langle\alpha^{-a}, \alpha^{b-2 n} \beta^{c}\right\rangle=\left\langle\alpha^{a}, \alpha^{b^{\prime}} \beta^{c}\right\rangle$.

\section{Finite-sheeted Covering maps over Klein Bottle}

In Section 2 we introduced pointed covering maps $f_{(p, q, r)}:\left(K, y_{0}\right) \rightarrow$ $\left(K, y_{0}\right), p \neq 0, r$ odd. Now, we will consider covering maps $f:\left(\mathbb{T}^{2}, x_{0}\right) \rightarrow$ $\left(K, y_{0}\right)$.

Each monomorphism $h_{A}: \mathbb{Z}^{2} \rightarrow G$ is of the form

$$
\begin{aligned}
& h_{A}(\alpha)=\alpha^{m} \beta^{2 n}, \\
& h_{A}\left(\beta^{2}\right)=\alpha^{k} \beta^{2 l}
\end{aligned}
$$

or equivalently,

$$
h_{A}\left(z_{1}, z_{2}\right)=\alpha^{m z_{1}+k z_{2}} \beta^{2\left(n z_{1}+l z_{2}\right)}=\left(m z_{1}+k z_{2}, 2\left(n z_{1}+l z_{2}\right)\right),
$$

where $A=\left[\begin{array}{ll}m & k \\ n & l\end{array}\right] \in M_{2}(\mathbb{Z}), \operatorname{det} A \neq 0$.

Let $A: \mathbb{R}^{2} \rightarrow \mathbb{R}^{2}, A=\left[\begin{array}{cc}m & k \\ n & l\end{array}\right] \in M_{2}(\mathbb{Z}), \operatorname{det} A \neq 0$. Then $A \alpha=\alpha^{m} \beta^{2 n} A$ and $A \beta^{2}=\alpha^{k} \beta^{2 l} A$. Thus $A: \mathbb{R}^{2} \rightarrow \mathbb{R}^{2}$ is a lifting of a map $f_{A}: \mathbb{T}^{2} \rightarrow K$ such that $f\left(x_{0}\right)=\left(y_{0}\right)$ and $f_{\#}=h_{A} \cdot f_{A}$ is a pointed $s$-sheeted covering map over $K$, where $s=2|\operatorname{det} A|$. Note that $f_{I}: \mathbb{T}^{2} \rightarrow K$, where $I$ is the identity matrix, is the basic 2 -sheeted covering map of $\mathbb{T}^{2}$ over $K$, i.e., $f_{I}=\delta$. 
Recall that each integral matrix $A=\left[\begin{array}{cc}m & k \\ n & l\end{array}\right]$, $\operatorname{det} A \neq 0$, defines an $s$ sheeted covering homomorphism $f^{A}:\left(\mathbb{T}^{2}, x_{0}\right) \rightarrow\left(\mathbb{T}^{2}, x_{0}\right)$, where $s=|\operatorname{det} A|$ (see $[3, \S 2])$. Note that $f_{A}=\delta f^{A}$, i.e., each $s$-sheeted covering map $f_{A}$ : $\left(\mathbb{T}^{2}, x_{0}\right) \rightarrow\left(K, y_{0}\right), s=2|\operatorname{det} A|$, can be represented as the composition of an $\frac{s}{2}$-sheeted covering homomorphism $f^{A}:\left(\mathbb{T}^{2}, x_{0}\right) \rightarrow\left(\mathbb{T}^{2}, x_{0}\right)$ and the basic 2-sheeted covering map $\delta:\left(\mathbb{T}^{2}, x_{0}\right) \rightarrow\left(K, y_{0}\right)$.

Let $f:(X, x) \rightarrow\left(K, y_{0}\right)$ be a pointed $s$-sheeted covering map. According to the classical classification theorem of covering maps, $H=f_{\#}\left(\pi_{1}(X, x)\right)$ is an $s$-index subgroup of $\pi_{1}\left(K, y_{0}\right)=G$. It follows from Proposition 3.4 that there are integers $a, b$ and $c$ such that $a, c \in \mathbb{N}, 0 \leq b<a, a c=s$, and $H=\left\langle\alpha^{a}, \alpha^{b} \beta^{c}\right\rangle$.

$f$ is pointed equivalent to

(1) $f_{(a, b, c)}:\left(K, y_{0}\right) \rightarrow\left(K, y_{0}\right)$, if $c$ is odd;

(2) $f_{A}:\left(\mathbb{T}^{2}, x_{0}\right) \rightarrow\left(K, y_{0}\right), A=\left[\begin{array}{ll}a & b \\ 0 & \frac{c}{2}\end{array}\right]$, if $c$ is even.

In the unpointed case, according to the considerations about conjugacy classes of $\left\langle\alpha^{a}, \alpha^{b} \beta^{c}\right\rangle$ in Section $3, f$ is equivalent to

(1) $f_{\left(a, b^{\prime}, c\right)}: K \rightarrow K, 0 \leq b^{\prime}<a$, if $a$ and $c$ are odd;

(2) $f_{\left(a, b^{\prime}, c\right)}: K \rightarrow K, 0 \leq b^{\prime}<a, b-b^{\prime}$ is even, if $a$ is even and $c$ is odd;

(3) $f_{A}: \mathbb{T}^{2} \rightarrow K, A=\left[\begin{array}{cc}a & b^{\prime} \\ 0 & \frac{c}{2}\end{array}\right], b^{\prime}$ equals $b$ or $a-b$, if $c$ is even.

Proposition 4.1. Let $(X, x)$ be a pointed Klein bottle weak solenoidal space. Then $(X, x)$ is pointed homeomorphic to a $(\Sigma(\boldsymbol{p}, \boldsymbol{q}, \boldsymbol{r}), *)$, where $p_{i}, r_{i}$ are positive and $0 \leq q_{i}<p_{i}$ for each $i$.

Proof. Let $\left\{\left(K, x_{i}\right), g_{i i+1}, \mathbb{N}\right\}$ be a pointed Klein bottle weak solenoidal sequence such that $(X, x)=\lim \left\{\left(K, x_{i}\right), g_{i i+1}, \mathbb{N}\right\}$. Since $K$ is homogenous there is a homeomorphism $h_{1}:\left(K, x_{1}\right) \rightarrow\left(K, y_{0}\right)$. By the induction for each $i \in \mathbb{N}$ we will find integers $p_{i}, q_{i}, r_{i}, p_{i}, r_{i} \in \mathbb{N}, r_{i}$ odd, $0 \leq q_{i}<p_{i}$ and a homeomorphism $h_{i+1}:\left(K, x_{i+1}\right) \rightarrow\left(K, y_{0}\right)$ such that $h_{i} g_{i i+1}=f_{\left(p_{i}, q_{i}, r_{i}\right)} h_{i+1}$. Let $i=1$. Since $h_{1} g_{12}:\left(K, x_{2}\right) \rightarrow\left(K, y_{0}\right)$ is a pointed covering map, there are positive integers $p_{1}, r_{1}, r_{1}$ odd, an integer $q_{1}, 0 \leq q_{1}<p_{1}$, and a pointed homeomorphism $h_{2}:\left(K, x_{2}\right) \rightarrow\left(K, y_{0}\right)$ such that $h_{1} g_{12}=f_{\left(p_{1}, q_{1}, r_{1}\right)} h_{2}$. Let us assume that homeomorphisms $h_{2}, \ldots, h_{n}$ and integers $p_{1}, p_{2}, \ldots, p_{n-1}$, $q_{1}, q_{2}, \ldots, q_{n-1}, r_{1}, r_{2}, \ldots, r_{n-1}$ with required properties are defined. Since $h_{n} g_{n n+1}:\left(K, x_{n+1}\right) \rightarrow\left(K, y_{0}\right)$ is a pointed covering map there are positive integers $p_{n}, r_{n}, r_{n}$ odd, an integer $q_{n}, 0 \leq q_{n}<p_{n}$, and a pointed homeomorphism $h_{n+1}:\left(K, x_{n+1}\right) \rightarrow\left(K, y_{0}\right)$ such that $h_{n} g_{n n+1}=f_{\left(p_{n}, q_{n}, r_{n}\right)} h_{n+1}$. This completes the inductive step. Now, pointed homeomorphisms $h_{n}:\left(K, x_{n}\right) \rightarrow$ $\left(K, y_{0}\right)$ induce a desired pointed homeomorphism $h:(X, x) \rightarrow(\Sigma(\boldsymbol{p}, \boldsymbol{q}, \boldsymbol{r}), *)$. 
In the sequel we will consider only pointed Klein bottle weak solenoidal spaces $(\Sigma(\boldsymbol{p}, \boldsymbol{q}, \boldsymbol{r}), *)$, where the sequences $\boldsymbol{p}=\left(p_{i}\right)$, and $\boldsymbol{r}=\left(r_{i}\right)$ consist of positive integers.

\section{Pull-Back diagrams}

The proof of the following proposition is very simple, so we omit it.

Proposition 5.1. Let $L$ be an arbitrary group, let $M, N$ be subgroups of $L$ and let $h: L \rightarrow L$ be a homomorphism. A function $\phi: L / M \rightarrow L / N$ defined by $\phi(M g)=N h(g)$ is a well-defined injection if and only if $M=h^{-1}(N)$.

Proposition 5.2. Let $h_{(p, q, r)}: G \rightarrow G$ be a monomorphism and let $H_{i}=$ $\left\langle\alpha^{a_{i}}, \alpha^{b_{i}} \beta^{c_{i}}\right\rangle, a_{i}, c_{i} \in \mathbb{N}, b_{i} \in \mathbb{Z}, i=1,2$, be subgroups of $G, c_{2}$ even. $A$ function $\phi: G / H_{1} \rightarrow G / H_{2}$ defined by $\phi\left(H_{1} g\right)=H_{2} h_{(p, q, r)}(g)$ is a welldefined bijection if and only if

(i) $G C D\left(p, a_{2}, r b_{2}\right)=1, G C D\left(c_{2}, r\right)=1$;

(ii) $c_{1}=d c_{2}, a_{2}=d a_{1}$, where $d=G C D\left(p, a_{2}\right)$;

(iii) $p^{\prime} b_{1} \equiv r b_{2}\left(\bmod a_{1}\right)$, where $p=d p^{\prime}$.

Proof. First note that $p \neq 0, r$ odd and $c\left(H_{i}\right)=c_{i}, i=1,2$. Let $\phi$ : $G / H_{1} \rightarrow G / H_{2}, \phi\left(H_{1} g\right)=H_{2} h_{(p, q, r)}(g)$, be a well-defined bijection. Then $a_{1} c_{1}=a_{2} c_{2}$. There are integers $m, n \in \mathbb{Z}$ such that $\phi\left(H_{1} \alpha^{m} \beta^{n}\right)=H_{2} \beta$, i.e., $h_{(p, q, r)}\left(\alpha^{m} \beta^{n}\right) \beta^{-1} \in H_{2}$. Hence $p r_{2}\left(\alpha^{p m}\left(\alpha^{q} \beta^{r}\right)^{n} \beta^{-1}\right)=n r-1 \in c_{2} \mathbb{Z}$, which implies $G C D\left(c_{2}, r\right)=1 . h_{(p, q, r)}\left(H_{1}\right) \subseteq H_{2}$ implies $h_{(p, q, r)}\left(\alpha^{b_{1}} \beta^{c_{1}}\right)=$ $\alpha^{p b_{1}}\left(\alpha^{q} \beta^{r}\right)^{c_{1}} \in H_{2}$ and consequently $r c_{1} \in c_{2} \mathbb{Z}$. Since $G C D\left(c_{2}, r\right)=1, c_{2}$ divides $c_{1}$ and $c_{1}$ is also even. Let $d^{\prime} \in \mathbb{Z}$ be such that $c_{1}=d^{\prime} c_{2}$. Then $a_{2}=d^{\prime} a_{1}$. There are integers $k, l \in \mathbb{Z}, 0 \leq k<a_{1}, 0 \leq l<c_{1}$, such that $\phi\left(H_{1} \alpha^{k} \beta^{l}\right)=$ $H_{2} \alpha$, i.e., $h_{(p, q, r)}\left(\alpha^{k} \beta^{l}\right) \alpha^{-1} \in H_{2}$. Hence $p r_{2}\left(\alpha^{p k}\left(\alpha^{q} \beta^{r}\right)^{l} \alpha^{-1}\right)=r l \in$ $c_{2} \mathbb{Z}$, which implies $l \equiv 0\left(\bmod c_{2}\right)$. Let $l=c_{2} l^{\prime}, l^{\prime} \in \mathbb{Z}$. We get $\phi\left(H_{1} \alpha^{k} \beta^{l}\right)=\phi\left(H_{1} \alpha^{k} \beta^{c_{2} l^{\prime}}\right)=H_{2} \alpha^{p k}\left(\alpha^{q} \beta^{r}\right)^{c_{2} l^{\prime}}=H_{2} \alpha^{p k} \beta^{c_{2} r l^{\prime}}=H_{2} \alpha$, which implies $\alpha^{p k-1} \beta^{c_{2} r l^{\prime}} \in H_{2}$. Since $\alpha^{p k-1} \beta^{c_{2} r l^{\prime}}=\alpha^{p k-b_{2} r l^{\prime}-1}\left(\alpha^{b_{2}} \beta^{c_{2}}\right)^{r l^{\prime}}$ it follows $\alpha^{p k-b_{2} r l^{\prime}-1} \in H_{2}$ and thus $G C D\left(p, a_{2}, r b_{2}\right)=1$. This proves (i).

Put $G C D\left(p, a_{2}\right)=d, a_{2}=d a^{\prime}$ and $p=d p^{\prime}$. Note $G C D\left(p^{\prime}, a^{\prime}\right)=1$. Since $h_{(p, q, r)}\left(\alpha^{a_{1}}\right)=\alpha^{p a_{1}} \in H_{2}$ and $h_{(p, q, r)}\left(\alpha^{b_{1}} \beta^{c_{1}}\right)=\alpha^{p b_{1}}\left(\alpha^{q} \beta^{r}\right)^{c_{1}}=$ $\alpha^{p b_{1}} \beta^{r c_{1}} \in H_{2}$, there are integers $w, u, v$ such that $\alpha^{p a_{1}}=\alpha^{w a_{2}}, \alpha^{p b_{1}} \beta^{r c_{1}}=$ $\alpha^{u a_{2}}\left(\alpha^{b_{2}} \beta^{c_{2}}\right)^{v}$, i.e., $p a_{1}=w a_{2}, p b_{1}=u a_{2}+v b_{2}$ and $r c_{1}=c_{2} v$. This implies $a_{2}=d a^{\prime} \mid p^{\prime} d a_{1}$, i.e., $a^{\prime} \mid a_{1}$ and consequently $d^{\prime} \mid d$. On the other hand $p b_{1}=u a_{2}+r d^{\prime} b_{2}$, i.e., $d \mid u a^{\prime} d+r d^{\prime} b_{2}$. Since $G C D\left(r b_{2}, d\right)=1$ we conclude $d \mid d^{\prime}$. Hence $d=d^{\prime}$ and $a_{2}=d a_{1}, a^{\prime}=a_{1}, c_{1}=d c_{2}$, which proves (ii).

Now we get $p^{\prime} d b_{1}=u a^{\prime} d+r d b_{2}$ or $p^{\prime} b_{1} \equiv r b_{2}\left(\bmod a^{\prime}\right)$, which proves (iii). 
Let us assume that the conditions (i), (ii) and (iii) are fulfilled and let us prove that $\phi: G / H_{1} \rightarrow G / H_{2}, \phi\left(H_{1} g\right)=H_{2} h_{(p, q, r)}(g)$, is a welldefined bijection. Since $\left[\dot{G}: H_{1}\right]=a_{1} c_{1}=a_{2} c_{2}=\left[\dot{G}: H_{2}\right]$, according to Proposition 5.1 it is enough to prove $h_{(p, q, r)}^{-1}\left(H_{2}\right)=H_{1}$. Note that $h_{(p, q, r)}\left(\alpha^{a_{1}}\right)=\alpha^{p a_{1}}=\alpha^{p^{\prime} a_{2}} \in H_{2}$ and $h_{(p, q, r)}\left(\alpha^{b_{1}} \beta^{c_{1}}\right)=\alpha^{p b_{1}}\left(\alpha^{q} \beta^{r}\right)^{c_{1}}=$ $\alpha^{p b_{1}} \beta^{r c_{1}}=\alpha^{p b_{1}} \beta^{r d c_{2}}=\alpha^{p^{\prime} d b_{1}-b_{2} r d}\left(\alpha^{b_{2}} \beta^{c_{2}}\right)^{r d}$. Since $d\left(p^{\prime} b_{1}-b_{2} d\right)=a_{2} k$ for some integer $k$ it follows that $h_{(p, q, r)}\left(\alpha^{b_{1}} \beta^{c_{1}}\right) \in H_{2}$. Thus $h_{(p, q, r)}\left(H_{1}\right) \subseteq H_{2}$ and $H_{1} \subseteq h_{(p, q, r)}^{-1}\left(H_{2}\right)$. Let us prove $h_{(p, q, r)}^{-1}\left(H_{2}\right) \subseteq H_{1}$. Let $\alpha^{n} \beta^{m} \in$ $h_{(p, q, r)}^{-1}\left(H_{2}\right)$. Then $h_{(p, q, r)}\left(\alpha^{n} \beta^{m}\right)=\alpha^{p n}\left(\alpha^{q} \beta^{r}\right)^{m} \in H_{2}$. Since $c_{2} \mid r m$ and $G C D\left(r, c_{2}\right)=1$ it follows $m=c_{2} m^{\prime}$ for some integer $m^{\prime}$. Then $\alpha^{p n}\left(\alpha^{q} \beta^{r}\right)^{m}=$ $\alpha^{p n}\left(\alpha^{q} \beta^{r}\right)^{c_{2} m^{\prime}}=\alpha^{p n} \beta^{c_{2} r m^{\prime}}=\alpha^{p n-b_{2} r m^{\prime}}\left(\alpha^{b_{2}} \beta^{c_{2}}\right)^{r m^{\prime}} \in H_{2}$. Hence $a_{2}=d a_{1} \mid$ $p^{\prime} d n-b_{2} r m^{\prime}$, which implies $d \mid m^{\prime}$. Let $m^{\prime}=d m^{\prime \prime}$ for some integer $m^{\prime \prime}$. We get $\alpha^{n} \beta^{m}=\alpha^{n} \beta^{c_{2} d m^{\prime \prime}}=\alpha^{n} \beta^{c_{1} m^{\prime \prime}}=\alpha^{n-b_{1}}\left(\alpha^{b_{1}} \beta^{c_{1}}\right)^{m^{\prime \prime}}$. Let $p n-b_{2} r m^{\prime}=a_{2} k$ for some integer $k$. We get $p^{\prime} n+p^{\prime} b_{1} m^{\prime \prime}-b_{2} r m^{\prime \prime}-p^{\prime} b_{1} m^{\prime \prime}=a_{1} k$ and there exists an integer $k^{\prime}$ such that $p^{\prime}\left(n-b_{1} m^{\prime \prime}\right)=a_{1} k^{\prime}$. Thus $a_{1} \mid p^{\prime}\left(n-b_{1} m^{\prime \prime}\right)$ and since $G C D\left(a_{1}, p^{\prime}\right)=1$, it follows $a_{1} \mid n-b_{1} m^{\prime \prime}$. Now we conclude that $\alpha^{n} \beta^{m}=\alpha^{n-b_{1} m^{\prime \prime}}\left(\alpha^{b_{1}} \beta^{c_{1}}\right)^{m^{\prime \prime}} \in H_{1}$.

It follows from Proposition 5.2(ii) that $c_{1}$ is also even.

Proposition 5.3. Let $h_{(p, q, r)}: G \rightarrow G$ be a monomorphism and let $H_{i}=$ $\left\langle\alpha^{a_{i}}, \alpha^{b_{i}} \beta^{c_{i}}\right\rangle, a_{i}, c_{i} \in \mathbb{N}, b_{i} \in \mathbb{Z}, i=1,2$, be subgroups of $G, c_{1}, c_{2}$ odd. $A$ function $\phi: G / H_{1} \rightarrow G / H_{2}$ defined by $\phi\left(H_{1} g\right)=H_{2} h_{(p, q, r)}(g)$ is a welldefined bijection if and only if

(i) $G C D\left(p, a_{2}\right)=1, G C D\left(c_{2}, r\right)=1$;

(ii) $c_{1}=c_{2}, a_{2}=a_{1}$;

(iii) $p b_{1} \equiv-q+b_{2}\left(\bmod a_{2}\right)$.

Proof. Let $\phi: G / H_{1} \rightarrow G / H_{2}, \phi\left(H_{1} g\right)=H_{2} h_{(p, q, r)}(g)$, be a well-defined bijection. Then $a_{1} c_{1}=a_{2} c_{2}$. There are integers $m, n \in \mathbb{Z}$ such that $\phi\left(H_{1} \alpha^{m} \beta^{n}\right)=H_{2} \beta$, i.e., $h_{(p, q, r)}\left(\alpha^{m} \beta^{n}\right) \beta^{-1} \in H_{2}$. Hence $p r_{2}\left(\alpha^{p m}\left(\alpha^{q} \beta^{r}\right)^{n} \beta^{-1}\right)=n r-1 \in c_{2} \mathbb{Z}$, which implies $\operatorname{GCD}\left(c_{2}, r\right)=1$. $h_{(p, q, r)}\left(H_{1}\right) \subseteq H_{2}$ implies $h_{(p, q, r)}\left(\alpha^{a_{1}}\right)=\alpha^{p a_{1}} \in H_{2}$ and $h_{(p, q, r)}\left(\alpha^{b_{1}} \beta^{c_{1}}\right)=$ $\alpha^{p b_{1}}\left(\alpha^{q} \beta^{r}\right)^{c_{1}}=\alpha^{p b_{1}+q} \beta^{r c_{1}} \in H_{2}$. Thus $p a_{1}=n a_{2}$ for some $n \in \mathbb{Z}$ and $r c_{1} \in c_{2} \mathbb{Z}$. Since $\operatorname{GCD}\left(c_{2}, r\right)=1, c_{2}$ divides $c_{1}$. Let $d \in \mathbb{Z}$ be such that $c_{1}=d c_{2}$. Then $a_{2}=d a_{1}$ and $d$ divides $p$. There are integers $u$ and $v$ such that $\alpha^{p b_{1}+q} \beta^{r c_{1}}=\left(\alpha^{a_{2}}\right)^{u}\left(\alpha^{b_{2}} \beta^{c_{2}}\right)^{v}$. First note that $v$ is odd and $\alpha^{p b_{1}+q} \beta^{r c_{1}}=\alpha^{a_{2} u} \alpha^{b_{2}} \beta^{c_{2} v}=\alpha^{a_{2} u+b_{2}} \beta^{c_{2} v}$ and consequently $v=r d$ and $p b_{1}+q=a_{2} u+b_{2}$, which implies $p b_{1} \equiv-q+b_{2}\left(\bmod a_{2}\right)$. This proves (iii).

Note that $G C D\left(p, a_{2}\right)$ divides $b_{2}-q$. On the other hand there are integers $k, l \in \mathbb{Z}, 0 \leq k<a_{1}, 0 \leq l<c_{1}$, such that $\phi\left(H_{1} \alpha^{k} \beta^{l}\right)=$ $H_{2} \alpha$, i.e., $h_{(p, q, r)}\left(\alpha^{k} \beta^{l}\right) \alpha^{-1} \in H_{2}$. Hence $p r_{2}\left(\alpha^{p k}\left(\alpha^{q} \beta^{r}\right)^{l} \alpha^{-1}\right)=r l \in$ 
$c_{2} \mathbb{Z}$, which implies $l \equiv 0\left(\bmod c_{2}\right)$. Let $l=c_{2} l^{\prime}, l^{\prime} \in \mathbb{Z}$. We consider two cases: $l$ is even and $l$ is odd. Let $l$ be even. Then $l^{\prime}$ is even and $\alpha^{p k}\left(\alpha^{q} \beta^{r}\right)^{l} \alpha^{-1}=\alpha^{p k-1} \beta^{r c_{2} l^{\prime}}=\alpha^{p k-1}\left(\alpha^{b_{2}} \beta^{c_{2}}\right)^{r l^{\prime}} \in H_{2}$. Thus $G C D\left(p, a_{2}\right)=1$. Let $l$ be odd. Then $l^{\prime}$ is odd and $\alpha^{p k}\left(\alpha^{q} \beta^{r}\right)^{l} \alpha^{-1}=$ $\alpha^{p k} \alpha^{q} \beta^{r l} \alpha^{-1}=\alpha^{p k+q+1} \beta^{r c_{2} l^{\prime}}=\alpha^{p k+q+1-b_{2}}\left(\alpha^{b_{2}} \beta^{c_{2}}\right)^{r l^{\prime}} \in H_{2}$, which implies $G C D\left(p, a_{2}, b_{2}-q\right)=1$. Since $G C D\left(p, a_{2}\right)$ divides $b_{2}-q$, it follows that $G C D\left(p, a_{2}\right)=1$. This proves (i).

$G C D\left(p, a_{2}\right)=1$ implies $d=1$. Hence $a_{2}=a_{1}$ and $c_{2}=c_{1}$, which proves (ii).

Let us assume that the conditions (i), (ii) and (iii) are fulfilled and let us prove that $\phi: G / H_{1} \rightarrow G / H_{2}, \phi\left(H_{1} g\right)=H_{2} h_{(p, q, r)}(g)$, is a well-defined bijection. Since $\left[G: H_{1}\right]=a_{1} c_{1}=a_{2} c_{2}=\left[G: H_{2}\right]$, according to Proposition 5.1 it is enough to prove $h_{(p, q, r)}^{-1}\left(H_{2}\right)=H_{1}$. Note that $h_{(p, q, r)}\left(\alpha^{a_{1}}\right)=$ $\alpha^{p a_{1}}=\alpha^{p a_{2}} \in H_{2}$ and $h_{(p, q, r)}\left(\alpha^{b_{1}} \beta^{c_{1}}\right)=\alpha^{p b_{1}}\left(\alpha^{q} \beta^{r}\right)^{c_{1}}=\alpha^{p b_{1}+q} \beta^{r c_{1}}=$ $\alpha^{p b_{1}} \beta^{r c_{2}}=\alpha^{p b_{1}+q-b_{2}}\left(\alpha^{b_{2}} \beta^{c_{2}}\right)^{r}$. Since $p b_{1} \equiv-q+b_{2}\left(\bmod a_{2}\right)$ it follows $h_{(p, q, r)}\left(\alpha^{b_{1}} \beta^{c_{1}}\right) \in H_{2}$. Thus $h_{(p, q, r)}\left(H_{1}\right) \subseteq H_{2}$ and $H_{1} \subseteq h_{(p, q, r)}^{-1}\left(H_{2}\right)$. Let us prove $h_{(p, q, r)}^{-1}\left(H_{2}\right) \subseteq H_{1}$. Let $\alpha^{n} \beta^{m} \in h_{(p, q, r)}^{-1}\left(H_{2}\right)$. Then $h_{(p, q, r)}\left(\alpha^{n} \beta^{m}\right)=$ $\alpha^{p n}\left(\alpha^{q} \beta^{r}\right)^{m} \in H_{2}$. Since $c_{2} \mid r m$ and $G C D\left(r, c_{2}\right)=1$ it follows $m=c_{2} m^{\prime}=$ $c_{1} m^{\prime}$ for some even integer $m^{\prime}$. We consider two cases: $m$ is even and $m$ is odd. Let $m$ be even. Then $m^{\prime}$ is even and $h_{(p, q, r)}\left(\alpha^{n} \beta^{m}\right)=\alpha^{p n}\left(\alpha^{q} \beta^{r}\right)^{m}=$ $\alpha^{p n} \beta^{r c_{2} m^{\prime}}=\alpha^{p n}\left(\alpha^{b_{2}} \beta^{c_{2}}\right)^{r m^{\prime}} \in H_{2}$. Thus $p n=a_{2} n^{\prime}=a_{1} n^{\prime}$, for some integer $n^{\prime}$. Since $G C D\left(p, a_{2}\right)=G C D\left(p, a_{1}\right)=1$ it follows $n=a_{1} n^{\prime \prime}$ for some integer $n^{\prime \prime}$. Hence $\alpha^{n} \beta^{m}=\alpha^{a_{1} n^{\prime \prime}} \beta^{c_{2} m^{\prime}}=\alpha^{a_{1} n^{\prime \prime}}\left(\alpha^{b_{2}} \beta^{c_{2}}\right)^{m^{\prime}}=\alpha^{a_{1} n^{\prime \prime}}\left(\alpha^{b_{1}} \beta^{c_{1}}\right)^{m^{\prime}} \in H_{1}$. Let $m$ be odd. Then $m^{\prime}$ is odd and $h_{(p, q, r)}\left(\alpha^{n} \beta^{m}\right)=\alpha^{p n}\left(\alpha^{q} \beta^{r}\right)^{m}=$ $\alpha^{p n+q} \beta^{r c_{2} m^{\prime}}=\alpha^{p n+q-b_{2}}\left(\alpha^{b_{2}} \beta^{c_{2}}\right)^{r m^{\prime}} \in H_{2}$. Thus $p n+q-b_{2}=a_{2} n^{\prime}$ for some integer $n^{\prime}$, i.e., $p n \equiv-q+b_{2}\left(\bmod a_{2}\right)$. Since $p b_{1} \equiv-q+b_{2}\left(\bmod a_{2}\right)$ and $G C D\left(p, a_{2}\right)=1$, it follows $n \equiv b_{1}\left(\bmod a_{2}\right)$. Thus, $n=b_{1}+a_{2} n^{\prime \prime}=b_{1}+a_{1} n^{\prime \prime}$ for some integer $n^{\prime \prime}$. We get $\alpha^{n} \beta^{m}=\alpha^{b_{1}+a_{1} n^{\prime \prime}} \beta^{c_{2} m^{\prime}}=\alpha^{a_{1} n^{\prime \prime}}\left(\alpha^{b_{1}} \beta^{c_{1}}\right)^{m^{\prime}} \in H_{1}$, which completes the proof.

Let

$$
\begin{aligned}
& \left(X^{\prime}, x^{\prime}\right) \stackrel{g^{\prime}}{\longleftarrow}(X, x) \\
& f^{\prime} \downarrow \quad \downarrow f \\
& \left(Y^{\prime}, y^{\prime}\right) \overleftarrow{g^{\prime \prime}}(Y, y)
\end{aligned}
$$

be a commutative diagram, where $f$ and $f^{\prime}$ are pointed covering maps and all four spaces are pathwise connected. Let $L=\pi_{1}(Y, y), L^{\prime}=\pi_{1}\left(Y^{\prime}, y^{\prime}\right)$, $M=f_{\#}\left(\pi_{1}(X, x)\right), M^{\prime}=f_{\#}^{\prime}\left(\pi_{1}\left(X^{\prime}, x^{\prime}\right)\right)$ and let $\varphi: L / M \rightarrow L^{\prime} / M^{\prime}$ be a function defined by $\varphi(M u)=M^{\prime} g_{\#}^{\prime \prime}(u)$. According to [4, Lemma 10] diagram $(*)$ is a pull-back diagram if and only if $\varphi$ is a bijection. This fact together with Propositions 5.2 and 5.3 implies two following corollaries. 
Corollary 5.4. Let $p, r, a, d$ be positive integers, $r$ odd, and let $f_{(p, q, r)}$ : $\left(K, y_{0}\right) \rightarrow\left(K, y_{0}\right), f_{A}:\left(\mathbb{T}^{2}, x_{0}\right) \rightarrow\left(K, y_{0}\right), f_{B}:\left(\mathbb{T}^{2}, x_{0}\right) \rightarrow\left(K, y_{0}\right)$ be pointed covering maps, where $A=\left[\begin{array}{lr}a & b_{1} \\ 0 & d\end{array}\right]$ and $B=\left[\begin{array}{cc}a & b_{2} \\ 0 & d\end{array}\right]$. If $G C D(p, a)=$ $G C D(d, r)=1$ and $p b_{1} \equiv r b_{2}(\bmod a)$, then $f_{B}, f_{(p, q, r)}, f_{A}$ can be completed to a pull-back diagram

$$
\begin{array}{lrr}
\left(\mathbb{T}^{2}, x_{0}\right) & \stackrel{f}{\longleftarrow} & \left(\mathbb{T}^{2}, x_{0}\right) \\
f_{B} \downarrow & & \downarrow f_{A} \\
\left(K, y_{0}\right) & \longleftarrow & \left(K, y_{0}\right)
\end{array}
$$

Furthermore, $f:\left(\mathbb{T}^{2}, x_{0}\right) \rightarrow\left(\mathbb{T}^{2}, x_{0}\right)$ is a covering map and $f_{\#}$ is represented by an integral matrix $\left[\begin{array}{cc}p & \frac{p b_{1}-b_{2} r}{a} \\ 0 & r\end{array}\right]$.

Note that $f:\left(\mathbb{T}^{2}, x_{0}\right) \rightarrow\left(\mathbb{T}^{2}, x_{0}\right)$ from Corollary 5.4 is a covering homomorphism (represented by the matrix $\left[\begin{array}{ll}p & \frac{p b_{1}-b_{2} r}{a} \\ 0 & r\end{array}\right]$ ) if and only if $q=0$.

Corollary 5.5. Let $p, r, a, c$ be positive integers, $r$ and $c$ odd, and let $f_{\left(a, b_{1}, c\right)}:\left(K, y_{0}\right) \rightarrow\left(K, y_{0}\right), f_{\left(a, b_{2}, c_{2}\right)}:\left(K, y_{0}\right) \rightarrow\left(K, y_{0}\right), f_{(p, q, r)}:\left(K, y_{0}\right) \rightarrow$ $\left(K, y_{0}\right)$ be pointed covering maps. If $G C D(p, a)=G C D(c, r)=1$ and $p b_{1} \equiv$ $b_{2}-q(\bmod a)$, then $f_{\left(a, b_{2}, c_{2}\right)}, f_{(p, q, r)}, f_{\left(a, b_{2}, c_{2}\right)}$ can be completed to a pull-back diagram

$$
\begin{array}{ccc}
\left(K, y_{0}\right) & \stackrel{f}{\longleftarrow} & \left(K, y_{0}\right) \\
f_{\left(a, b_{2}, c\right)} \downarrow & & \downarrow f_{\left(a, b_{1}, c\right)} . \\
\left(K, y_{0}\right) & \longleftarrow & \left(K, y_{0}\right)
\end{array}
$$

Furthermore, $\quad f:\left(K, y_{0}\right) \rightarrow\left(K, y_{0}\right)$ is a covering map and $f_{\#}=$ $h_{\left(p, \frac{p b_{1}-b_{2}+q}{a}, r\right)}$.

\section{Pointed finite-SheEted COVERING MAPs OVer $(\Sigma(\boldsymbol{p}, \boldsymbol{q}, \boldsymbol{r}), *)$}

Let $\mathbb{N}_{k}$ denote a set $\{i \in \mathbb{N}: i \geq k\}$.

Definition 6.1. Let $a, c \in \mathbb{N}$. We say that

$$
\mathbf{b}_{(a, c)}=\left(b_{k}, b_{k+1}, \ldots, b_{n}, \ldots\right) \in\{0,1, \ldots, a-1\}^{\mathbb{N}_{k}}
$$

is an admissible sequence for $\Sigma(\boldsymbol{p}, \boldsymbol{q}, \boldsymbol{r})$ if for each $i \in \mathbb{N}_{k}$

(i) $G C D\left(a, p_{i}\right)=G C D\left(c, r_{i}\right)=1$;

(ii) $p_{i} b_{i+1} \equiv b_{i}-q_{i}(\bmod a)$, if $c$ is odd;

(iii) $p_{i} b_{i+1} \equiv r_{i} b_{i}(\bmod a)$, if $c$ is even. 
We consider two admissible sequences $\mathbf{b}_{(a, c)} \in\{0,1, \ldots, a-1\}^{\mathbb{N}_{k}}$, $\mathbf{b}_{\left(a^{\prime}, c^{\prime}\right)}^{\prime} \in\left\{0,1, \ldots, a^{\prime}-1\right\}^{\mathbb{N}_{k^{\prime}}}$ for $\Sigma(\boldsymbol{p}, \boldsymbol{q}, \boldsymbol{r})$ as equal, provided $a=a^{\prime}, c=c^{\prime}$ and there is an index $i^{*} \geq k, k^{\prime}$ such that $b_{i}=b_{i}^{\prime}$ for $i \geq i^{*}$.

Theorem 6.2. Let $(\Sigma(\boldsymbol{p}, \boldsymbol{q}, \boldsymbol{r}), *)$ be a pointed Klein bottle weak solenoidal space and let $s \in \mathbb{N}$. Then there is a bijection $F_{*}$ between the set of all admissible sequences $\mathbf{b}_{(a, c)}$ for $\Sigma(\boldsymbol{p}, \boldsymbol{q}, \boldsymbol{r})$, where ac $=s$, and the set of all equivalence classes of pointed s-sheeted covering maps $f_{*}:(X, x) \rightarrow(\Sigma(\boldsymbol{p}, \boldsymbol{q}, \boldsymbol{r}), *)$ with a connected total space. Moreover, if $F_{*}\left(\mathbf{b}_{(a, c)}\right)=\left[f_{*}\right]$, then $X$ is homeomorphic to a toroidal group if $c$ is even, while $X$ is homeomorphic to $\Sigma(\boldsymbol{p}, \boldsymbol{q}, \boldsymbol{r})$ if $c$ is odd.

Proof. Let $\mathbf{b}_{(a, c)} \in\{0,1, \ldots, a-1\}^{\mathbb{N}_{k}}$ be an admissible sequence for $\Sigma(\boldsymbol{p}, \boldsymbol{q}, \boldsymbol{r}), a c=s$. Depending on $c$ we will associate to $\mathbf{b}_{(a, c)}$ a pointed $s$ sheeted covering map $f_{*}:(X, x) \rightarrow(\Sigma(\boldsymbol{p}, \boldsymbol{q}, \boldsymbol{r}), *)$ with connected total space $X$ in the following manner.

1. $c$ is even. For each $i \in \mathbb{N}_{k}$ put $A_{i}=\left[\begin{array}{cc}a & b_{i} \\ 0 & \frac{c}{2}\end{array}\right]$ and let $f_{A_{i}}:\left(\mathbb{T}^{2}, x_{0}\right) \rightarrow$ $\left(K, y_{0}\right)$ be an $s$-sheeted covering map obtained by $A_{i}$. According to Proposition 5.4, $f_{A_{i}}, f_{\left(p_{i}, q_{i}, r_{i}\right)}, f_{A_{i+1}}$ can be completed to a pull-back diagram

$$
\begin{array}{lll}
\left(\mathbb{T}^{2}, x_{0}\right) & \stackrel{f_{i i+1}}{ } & \left(\mathbb{T}^{2}, x_{0}\right) \\
f_{A_{i}} \downarrow & & \downarrow f_{A_{i+1}} \\
\left(K, y_{0}\right) & f_{\left(p_{i}, q_{i}, r_{i}\right)} & \left(K, y_{0}\right)
\end{array}
$$

for each $i \geq k$, where $f_{i i+1}$ is a covering map and $f_{i i+1 \#}=\left[\begin{array}{cc}p_{i} & \frac{p b_{i+1}-b_{i} r}{a} \\ 0 & r_{i}\end{array}\right]$. Let $x_{i}=x_{0}$ for each $i, x=\left(x_{i}\right)$ and let $(X, x)$ be the inverse limit of a pointed inverse sequence $\boldsymbol{X}_{*}=\left\{\left(\mathbb{T}^{2}, x_{0}\right), f_{i i+1}, \mathbb{N}_{k}\right\}$. Note that $X$ is a torus solenoidal space, which is pointed homeomorphic to a pointed toroidal group $(A, x)$ obtained by matrices $\left[\begin{array}{cc}p_{i} & \frac{p b_{i+1}-b_{i} r}{a} \\ 0 & r_{i}\end{array}\right]$. Let $\boldsymbol{f}_{*}=\left\{f_{A_{i}}:\left(\mathbb{T}^{2}, x_{0}\right) \rightarrow\right.$ $\left.\left(K, y_{0}\right) \mid i \in \mathbb{N}_{k}\right\}: \boldsymbol{X}_{*} \rightarrow \boldsymbol{Y}_{*}=\left\{\left(K, y_{0}\right), f_{\left(p_{i}, q_{i}, r_{i}\right)}, \mathbb{N}\right\}$ be a mapping of pointed inverse sequences and let $f_{*}=\varliminf_{*} \boldsymbol{f}_{*}:(X, x) \rightarrow(\Sigma(\boldsymbol{p}, \boldsymbol{q}, \boldsymbol{r}), *)$.

2. $c$ is odd. For each $i \in \mathbb{N}_{k}$ let $f_{\left(a, b_{i}, c\right)}:\left(K, y_{0}\right) \rightarrow\left(K, y_{0}\right)$ be an $s$-sheeted covering map. According to Corollary 5.5, $f_{\left(a, b_{i}, c\right)}, f_{\left(p_{i}, q_{i}, r_{i}\right)}, f_{\left(a, b_{i+1}, c\right)}$ can be completed to a pull-back diagram

$$
\begin{array}{ccc}
\left(K, y_{0}\right) & f_{i} & \left(K, y_{0}\right) \\
f_{\left(a, b_{i}, c\right)} \downarrow & & \downarrow f_{\left(a, b_{i+1}, c\right)} \\
\left(K, y_{0}\right) & f_{\left(p_{i}, q_{i}, r_{i}\right)} & \left(K, y_{0}\right)
\end{array}
$$


for each $i \geq k$, where $f_{i}$ is a covering map and $f_{i \#}=h_{\left(p_{i}, \frac{p_{i} b_{i+1}-b_{i}+q_{i}}{a}, r_{i}\right)}$. Let $(X, x), x=*$, be the inverse limit of a pointed inverse sequence $\boldsymbol{X}_{*}=$ $\left\{\left(K, y_{0}\right), f_{i}, \mathbb{N}_{k}\right\}$. Note that $X$ is a Klein bottle weak solenoidal space. According to Proposition 4.1, $(X, *)$ is pointed homeomorphic to $\left(\Sigma\left(\boldsymbol{p}^{\prime}, \boldsymbol{q}^{\prime}, \boldsymbol{r}^{\prime}\right), *\right)$, where sequences $\boldsymbol{p}^{\prime}$ and $\boldsymbol{r}^{\prime}$ consist of positive integers. Moreover, there are homeomorphisms $h_{i}:\left(K, y_{0}\right) \rightarrow\left(K, y_{0}\right)$ such that $f_{\left(p_{i}^{\prime}, q_{i}^{\prime}, r_{i}^{\prime}\right)}, f_{i}, h_{i}, h_{i+1}$ form a commutative diagram

$$
\begin{array}{ccc}
\left(K, y_{0}\right) & f_{\left(p_{i}^{\prime}, q_{i}^{\prime}, r_{i}^{\prime}\right)} & \left(K, y_{0}\right) \\
h_{i} \downarrow & & \downarrow h_{i+1} \\
\left(K, y_{0}\right) & \longleftarrow & \left(K, y_{0}\right)
\end{array}
$$

for each $i$. Since $h_{i \#}$ are isomorphisms, i.e., $h_{i \#}=h_{\left(u_{i}, v_{i}, w_{i}\right)}$, where $u_{i}$ and $w_{i}$ are 1 or -1 , and $h_{\left(u_{i}, v_{i}, w_{i}\right)} h_{\left(p_{i}^{\prime}, q_{i}^{\prime}, r_{i}^{\prime}\right)}=h_{\left(p_{i}, \frac{p_{i} b_{i+1}-b_{i}+q_{i}}{a}, r_{i}\right)} h_{\left(u_{i+1}, v_{i+1}, w_{i+1}\right)}$ we get $u_{i} p_{i}^{\prime}=p_{i} u_{i+1}$ and $w_{i} r_{i}^{\prime}=r_{i} w_{i+1}$, which implies $p_{i}=p_{i}^{\prime}$ and $r_{i}=r_{i}^{\prime}$ for each $i$, which implies that $X$ is homeomorphic to $\Sigma(\boldsymbol{p}, \boldsymbol{q}, \boldsymbol{r})$. Let $\boldsymbol{f}_{*}=$ $\left\{f_{\left(a, b_{i}, c\right)}:\left(K, y_{0}\right) \rightarrow\left(K, y_{0}\right) \mid i \in \mathbb{N}_{k}\right\}: \boldsymbol{X}_{*} \rightarrow \boldsymbol{Y}_{*}=\left\{\left(K, y_{0}\right), f_{\left(p_{i}, q_{i}, r_{i}\right)}, \mathbb{N}\right\}$ be a mapping of pointed inverse sequences and let $f_{*}=\lim _{*}:(X, x) \rightarrow$ $(\Sigma(\boldsymbol{p}, \boldsymbol{q}, \boldsymbol{r}), *)$.

In both cases $f_{*}$ is a pointed $s$-sheeted covering map (see [5, Theorem 6 and Remark 1]). Now put $F_{*}\left(\mathbf{b}_{(a, c)}\right)=\left[f_{*}\right]$. We claim that $F_{*}$ is a bijection.

Claim 1. $F_{*}$ is an injection.

Let $F_{*}\left(\mathbf{b}_{(a, c)}\right)=F_{*}\left(\mathbf{b}_{\left(a^{\prime}, c^{\prime}\right)}\right), \mathbf{b}_{\left(a^{\prime}, c^{\prime}\right)} \in\left\{0,1, \ldots, a^{\prime}-1\right\}^{\mathbb{N}_{k^{\prime}}}, a c=a^{\prime} c^{\prime}=$ $s$. Then $\left[f_{*}\right]=F_{*}\left(\mathbf{b}_{(a, c)}\right)=F_{*}\left(\mathbf{b}_{\left(a^{\prime}, 2 c^{\prime}\right)}\right)=\left[f_{*}^{\prime}\right], f^{\prime}:\left(X^{\prime}, x^{\prime}\right) \rightarrow(\Sigma(\boldsymbol{p}, \boldsymbol{q}, \boldsymbol{r}), *)$. Let $\Phi_{*}$ be a bijection between the set of all pointed equivalence classes of $s$-sheeted covering maps $f:(X, x) \rightarrow(\Sigma(\boldsymbol{p}, \boldsymbol{q}, \boldsymbol{r}), *)$ and the set of all subprogroups of index $s$ of the fundamental progroup $\pi_{1}(\Sigma(\boldsymbol{p}, \boldsymbol{q}, \boldsymbol{r}), *)$ (see [5, Theorems 5 and 6]). Then $\Phi_{*}\left(\left[f_{*}\right]\right)=\left\{H_{i}, f_{\left(p_{i}, q_{i}, r_{i}\right) \#}, i \geq i_{1} \geq k\right\}=$ $\left\{\left\langle\alpha^{a}, \alpha^{b_{i}} \beta^{c}\right\rangle, h_{\left(p_{i}, q_{i}, r_{i}\right)}, i \geq i_{1} \geq k\right\}$, where each $H_{i}=\operatorname{Im} f_{A_{i} \#}=\left\langle\alpha^{a}, \alpha^{b_{i}} \beta^{c}\right\rangle$ if $c$ is even or each $H_{i}=\operatorname{Im} f_{\left(a, b_{i}, c\right) \#}=\left\langle\alpha^{a}, \alpha^{b_{i}} \beta^{c}\right\rangle$ if $c$ is odd. Analogously, $\Phi_{*}\left(\left[f_{*}^{\prime}\right]\right)=\left\{H_{i}^{\prime}, f_{\left(p_{i}, q_{i}, r_{i}\right) \#}, i \geq i_{1}^{\prime} \geq k^{\prime}\right\}$, where each $H_{i}^{\prime}=\operatorname{Im} f_{A_{i}^{\prime} \#}=$ $\left\langle\alpha^{a^{\prime}}, \alpha^{b_{i}^{\prime}} \beta^{c^{\prime}}\right\rangle$ if $c^{\prime}$ is even or each $H_{i}^{\prime}=\operatorname{Im} f_{\left(a^{\prime}, b_{i}^{\prime}, c^{\prime}\right) \#}=\left\langle\alpha^{a^{\prime}}, \alpha^{b_{i}^{\prime}} \beta^{c^{\prime}}\right\rangle$ if $c^{\prime}$ is odd. Since $\Phi_{*}\left(\left[f_{*}\right]\right)=\Phi_{*}\left(\left[f_{*}^{\prime}\right]\right)$ there is an $i^{*} \geq i_{1}, i_{1}^{\prime}$ such that $\left\langle\alpha^{a}, \alpha^{b_{i}} \beta^{c}\right\rangle=\left\langle\alpha^{a^{\prime}}, \alpha^{b_{i}^{\prime}} \beta^{c^{\prime}}\right\rangle$ for $i \geq i^{*}$. According to Proposition 3.4, $a=a^{\prime}$, $c=c^{\prime}, b_{i}=b_{i}^{\prime}$ for $i \geq i^{*}$ and consequently $\mathbf{b}_{(a, c)}=\mathbf{b}_{\left(a^{\prime}, c^{\prime}\right)}$.

Claim 2. $F_{*}$ is a surjection.

Let $g_{*}:(X, x) \rightarrow(\Sigma(\boldsymbol{p}, \boldsymbol{q}, \boldsymbol{r}), *)$ be a pointed $s$-sheeted covering map. Then there is a pointed inverse sequence $\boldsymbol{X}_{*}=\left\{\left(X_{i}, x_{i}\right), g_{i i+1}, \mathbb{N}_{k}\right\}$ and a mapping $\boldsymbol{g}_{*}=\left\{g_{i}:\left(X, x_{i}\right) \rightarrow\left(K, y_{0}\right) \mid i \in \mathbb{N}_{k}\right\}: \boldsymbol{X}_{*} \rightarrow$ $\boldsymbol{Y}_{*}=\left\{\left(K, y_{0}\right), f_{\left(p_{i}, q_{i}, r_{i}\right)}, \mathbb{N}\right\}$ of pointed inverse sequences such that each 
$g_{i}:\left(X, x_{i}\right) \rightarrow\left(K, y_{0}\right)$ is a pointed $s$-sheeted covering map with a connected total space, each

$$
\begin{aligned}
& \left(X_{i}, x_{i}\right) \quad \stackrel{g_{i i+1}}{\longleftarrow}\left(X_{i+1}, x_{i+1}\right) \\
& g_{i} \downarrow \quad \downarrow g_{i+1} \\
& \left(K, y_{0}\right) \underset{f_{\left(p_{i}, q_{i}, r_{i}\right)}}{\longleftarrow}\left(K, y_{0}\right)
\end{aligned}
$$

is a pull-back diagram, $(X, x)=\lim \boldsymbol{X}_{*}$ and $g_{*}=\lim _{*}$.

Since each $g_{i}:\left(X, x_{i}\right) \rightarrow\left(K, y_{0}\right)$ is a pointed $s$-sheeted covering map it follows that $H_{i}=\operatorname{Im} g_{i \#}=g_{i \#}\left(\pi_{1}\left(X_{i}, x_{i}\right)\right)$ is a subprogroup of index $s$ of $G$. Hence there are integers $a_{i}, c_{i} \in \mathbb{N}$ and $0 \leq b_{i}<a_{i}$ such that $H_{i}=\left\langle\alpha^{a_{i}}, \alpha^{b_{i}} \beta^{c_{i}}\right\rangle, a_{i} c_{i}=s$. Moreover, $\phi_{i}: G / H_{i+1} \rightarrow G / H_{i}$ defined by $\phi_{i}\left(H_{i+1} v\right)=H_{i} h_{\left(p_{i}, q_{i}, r_{i}\right)}(v)$ is a well-defined bijection for each $i$. We distinct two cases:

a) There is $i^{*} \geq k$ such that $c_{i^{*}}$ is even. According to Proposition 5.2, for each $i \geq i^{*}, c_{i}$ is even, $G C D\left(p_{i}, a_{i}, r_{i} b_{i}\right)=G C D\left(c_{i}, r_{i}\right)=1, c_{i+1}=d_{i} c_{i}, a_{i}=$ $d_{i} a_{i+1}$, and $p^{\prime} b_{i+1} \equiv r_{i} b_{i}\left(\bmod a_{i+1}\right)$, where $d_{i}=G C D\left(p_{i}, a_{i}\right)$ and $p_{i}=d_{i} p_{i}^{\prime}$. Note that for each $i \geq i^{*}, a_{i+1}$ divides $a_{i}$ if $d_{i}=G C D\left(p_{i}, a_{i}\right)>1$ or $a_{i+1}=a_{i}$ if $d_{i}=G C D\left(p_{i}, a_{i}\right)=1$. Since each positive number has only finite many divisors there are $k^{*} \geq i^{*}$ and positive numbers $a$ and $c, c$ even, such that for each $i \geq k^{*}, a_{i}=a, c_{i}=c, G C D\left(p_{i}, a\right)=G C D\left(c, r_{i}\right)=1$ and $p_{i} b_{i+1} \equiv r_{i} b_{i}$ $(\bmod a)$. Put $\mathbf{b}_{(a, c)}=\left(b_{k^{*}}, b_{k^{*}+1}, \ldots, b_{n}, \ldots\right) \in\{0,1, \ldots, a-1\}^{\mathbb{N}_{k^{*}}}$. Obviously, $\mathbf{b}_{(a, c)}$ is admissible for $(\Sigma(\boldsymbol{p}, \boldsymbol{q}, \boldsymbol{r}), *)$. Let $F_{*}\left(\mathbf{b}_{(a, c)}\right)=\left[f_{*}\right]$, where $f_{*}$ is obtained as in 1. Then $\Phi_{*}\left(\left[f_{*}\right]\right)=\left\{\left\langle\alpha^{a}, \alpha^{b_{i}} \beta^{c}\right\rangle, h_{\left(p_{i}, q_{i}, r_{i}\right)}, i \geq i_{1} \geq k^{*}\right\}=$ $\left\{\left\langle\alpha^{a}, \alpha^{b_{i}} \beta^{c}\right\rangle, h_{\left(p_{i}, q_{i}, r_{i}\right)}, i \geq i_{1} \geq k\right\}=\Phi_{*}\left(\left[g_{*}\right]\right)$, which implies $\left[f_{*}\right]=\left[g_{*}\right]$ and $F_{*}\left(\mathbf{b}_{(a, c)}\right)=\left[g_{*}\right]$.

b) Each $c_{i}$ is odd, $i \geq k$. According to Proposition 5.3, for each $i \geq k$, $G C D\left(p_{i}, a_{i}\right)=G C D\left(c_{i}, r_{i}\right)=1, c_{i+1}=c_{i}, a_{i+1}=a_{i}$ and $p_{i} b_{i+1} \equiv b_{i}-q_{i}$ $\left(\bmod a_{i}\right)$. Hence there are positive integers $a$ and $c, c$ odd, such that $G C D\left(p_{i}, a\right)=G C D\left(c, r_{i}\right)=1$ and $p_{i} b_{i+1} \equiv b_{i}-q_{i}(\bmod a)$. Put $\mathbf{b}_{(a, c)}=$ $\left(b_{k}, b_{k+1}, \ldots, b_{n}, \ldots\right) \in\{0,1, \ldots, a-1\}^{\mathbb{N}_{k}}$. Obviously $\mathbf{b}_{(a, c)}$ is admissible for $(\Sigma(\boldsymbol{p}, \boldsymbol{q}, \boldsymbol{r}), *)$. Let $F_{*}\left(\mathbf{b}_{(a, c)}\right)=\left[f_{*}\right]$, where $f_{*}$ is obtained as in 2. Then $\Phi_{*}\left(\left[f_{*}\right]\right)=\left\{\left\langle\alpha^{a}, \alpha^{b_{i}} \beta^{c}\right\rangle, h_{\left(p_{i}, q_{i}, r_{i}\right)}, i \geq i_{1}^{\prime} \geq k\right\}=\left\{\left\langle\alpha^{a}, \alpha^{b_{i}} \beta^{c}\right\rangle, h_{\left(p_{i}, q_{i}, r_{i}\right)}, i \geq\right.$ $\left.i_{1} \geq k\right\}=\Phi_{*}\left(\left[g_{*}\right]\right)$, which implies $\left[f_{*}\right]=\left[g_{*}\right]$ and $F_{*}\left(\mathbf{b}_{(a, c)}\right)=\left[g_{*}\right]$.

In both cases a) and b) we find an admissible sequence $\mathbf{b}_{(a, c)}$ such that $F_{*}\left(\mathbf{b}_{(a, c)}\right)=\left[g_{*}\right]$, which proves that $F_{*}$ is a surjection.

REMARK 6.3. Each Klein bottle weak solenoidal space $\Sigma(\boldsymbol{p}, \boldsymbol{q}, \boldsymbol{r})$ admits a pointed double-sheeted covering map with total space homeomorphic to the product $\Sigma(\boldsymbol{p}) \times \Sigma(\boldsymbol{r})$ of solenoids $\Sigma(\boldsymbol{p})$ and $\Sigma(\boldsymbol{r})$, obtained by sequences $\boldsymbol{p}$ and $r$ respectively. 
THEOREM 6.4. Let $n_{(a, c)}$ denote the total number of different admissible sequences $\mathbf{b}_{(a, c)}$ for $\Sigma(\boldsymbol{p}, \boldsymbol{q}, \boldsymbol{r}),(a, c) \in \mathbb{N} \times \mathbb{N}$ fixed. If $a=d_{1}^{\alpha_{1}} d_{2}^{\alpha_{2}} \cdots d_{m}^{\alpha_{m}}, d_{i}$ different primes, $\alpha_{i}$ positive integers, let $\left\{d_{i_{1}}, d_{i_{2}}, \ldots, d_{i_{n}}\right\} \subseteq\left\{d_{1}, \ldots, d_{m}\right\}$ be the set (possibly empty) of all prime divisors of a which divide infinitely many terms in the sequence $\boldsymbol{r}=\left(r_{i}\right)$. Then

$$
n_{(a, c)}=\left\{\begin{array}{ll}
\frac{a}{d_{i_{1}}^{\alpha_{i_{1}}} d_{i_{2}}^{\alpha_{i}} \cdots d_{i_{n}}^{\alpha_{i}}}, & c \text { even } \\
a, & c \text { odd }
\end{array} .\right.
$$

Proof. Let $k \in \mathbb{N}$ be an integer such that $G C D\left(a, p_{i}\right)=G C D\left(c, r_{i}\right)=$ $G C D\left(d, r_{i}\right)=1$ for each $i \geq k$ and each $d \in\left\{d_{1}, d_{2} \ldots, d_{m}\right\} \backslash\left\{d_{i_{1}}, d_{i_{2}}, \ldots, d_{i_{n}}\right\}$. We will define sequences

$$
\mathbf{b}_{(a, c)}^{j}=\left(b_{k}^{j}, b_{k+1}^{j}, \ldots, b_{n}^{j}, \ldots\right) \in\{0,1, \ldots, a-1\}^{\mathbb{N}_{k}}, j=0,1, \ldots, a-1,
$$

in the following way. If $c$ is even, let $x_{i} \in\{0,1, \ldots, a-1\}$ be a unique solution of a linear congruence $p_{i} x_{i} \equiv r_{i}(\bmod a), i \geq k$. If $c$ is odd, let $x_{i}, y_{i} \in$ $\{0,1, \ldots, a-1\}$ be unique solutions of linear congruences $p_{i} x_{i} \equiv 1(\bmod a)$ and $p_{i} y_{i} \equiv-q_{i}(\bmod a), i \geq k$. If $a=1$, we put $\mathbf{b}_{(1, c)}^{0}=(0,0, \ldots, 0, \ldots) \in$ $\{0\}^{\mathbb{N}_{k}}$. If $a \geq 2$, we put $\mathbf{b}_{(a, c)}^{j}=\left(j, b_{k+1}^{j}, \ldots, b_{n}^{j}, \ldots\right)$, where $b_{i+1}^{j} \equiv x_{i} b_{i}^{j}$ $(\bmod a), i \geq k$, if $c$ is even or $b_{i+1}^{j} \equiv x_{i} b_{i}^{j}+y_{i}(\bmod a), i \geq k$, if $c$ is odd. We claim that $\mathbf{b}_{(a, c)}^{j}$ are admissible for $\Sigma(\boldsymbol{p}, \boldsymbol{q}, \boldsymbol{r})$. If $c$ is even, we get $p_{i} b_{i+1}^{j} \equiv$ $p_{i} x_{i} b_{i}^{j} \equiv r_{i} b_{i}^{j}(\bmod a)$. If $c$ is odd, we get $p_{i} b_{i+1}^{j} \equiv p_{i} x_{i} b_{i}^{j}+p_{i} y_{i} \equiv b_{i}^{j}-q_{i}$ $(\bmod a)$. Hence, in both cases $\mathbf{b}_{(a, c)}^{j}$ are admissible for $\Sigma(\boldsymbol{p}, \boldsymbol{q}, \boldsymbol{r})$.

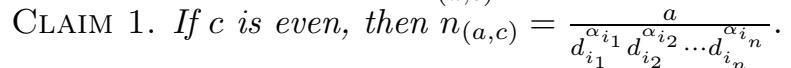

Note that $G C D\left(a, r_{i}\right)=G C D\left(a, x_{i}\right), i \geq k$, and $b_{k+n}^{j} \equiv\left(\prod_{i=k}^{k+n-1} x_{i}\right) j$ $(\bmod a)$ for an arbitrary $n \in \mathbb{N}$.

If $\left\{d_{i_{1}}, d_{i_{2}}, \ldots, d_{i_{n}}\right\}=\emptyset$, then $1=G C D\left(a, r_{i}\right)=G C D\left(a, x_{i}\right), i \geq k$. In this case $b_{k+n}^{j} \equiv b_{k+n}^{j^{\prime}}(\bmod a)$ implies $j \equiv j^{\prime}(\bmod a)$, which shows that all $\mathbf{b}_{(a, c)}^{j}, j=0,1, \ldots, a-1$, are different admissible sequences for $\Sigma(\boldsymbol{p}, \boldsymbol{q}, \boldsymbol{r})$.

Let $\left\{d_{i_{1}}, d_{i_{2}}, \ldots, d_{i_{n}}\right\} \neq \emptyset$. We claim that $\mathbf{b}_{(a, c)}^{j}=\mathbf{b}_{(a, c)}^{j^{\prime}}$ if and only if $j \equiv j^{\prime}\left(\bmod \frac{a}{d_{i_{1}}^{\alpha_{i_{1}}} d_{i_{2}}^{\alpha_{2}} \cdots d_{i_{n}}^{\alpha_{i_{n}}}}\right)$. Let $\mathbf{b}_{(a, c)}^{j}=\mathbf{b}_{(a, c)}^{j^{\prime}}$. Then there is $n_{0} \in \mathbb{N}$ such that $b_{k+n}^{j}=b_{k+n}^{j^{\prime}}$ for $n \geq n_{0}$. On the other hand we can choose large enough $n_{1} \geq n_{0}$ such that $\prod_{i=k}^{k+n_{1}-1} x_{i}=\lambda d_{i_{1}}^{l_{1}} d_{i_{2}}^{l_{2}} \cdots d_{i_{n}}^{l_{n}}$, where $\lambda \in \mathbb{N}$ and $l_{j} \geq \alpha_{i_{j}}$ for $j=1, \ldots, n$. Note that $d \nmid \lambda$ for each $d \notin\left\{d_{i_{1}}, d_{i_{2}}, \ldots, d_{i_{n}}\right\}$. Now, $b_{k+n_{1}}^{j} \equiv$ $b_{k+n_{1}}^{j^{\prime}}(\bmod a)$ implies $\lambda d_{i_{1}}^{l_{1}} d_{i_{2}}^{l_{2}} \cdots d_{i_{n}}^{l_{n}}\left(j-j^{\prime}\right) \equiv 0(\bmod a)$ and we conclude $j-j^{\prime} \equiv 0\left(\bmod \frac{a}{d_{i_{1}}^{\alpha_{i_{1}}} d_{i_{2}}^{\alpha_{i}} \cdots d_{i_{n}}^{\alpha_{i}}}\right)$. Let now $j-j^{\prime} \equiv 0\left(\bmod \frac{a}{d_{i_{1}}^{\alpha_{i_{1}}} d_{i_{2}}^{\alpha_{i_{2}} \cdots d_{i_{n}}{ }^{\alpha_{i}}{ }_{n}}}\right)$. 
Then $d_{i_{1}}^{\alpha_{i_{1}}} d_{i_{2}}^{\alpha_{i_{2}}} \cdots d_{i_{n}}^{\alpha_{i_{n}}}\left(j-j^{\prime}\right) \equiv 0(\bmod a)$. Choose large enough $n$ such that $\prod_{i=k}^{k+n-1} x_{i}=\lambda d_{i_{1}}^{l_{1}} d_{i_{2}}^{l_{2}} \cdots d_{i_{n}}^{l_{n}}$, where $\lambda \in \mathbb{N}$, and $l_{j} \geq \alpha_{i_{j}}$ for $j=1, \ldots, n$. Then $b_{k+n}^{j}-b_{k+n}^{j^{\prime}} \equiv \lambda d_{i_{1}}^{l_{1}} d_{i_{2}}^{l_{2}} \cdots d_{i_{n}}^{l_{n}}\left(j-j^{\prime}\right) \equiv 0(\bmod a)$. Hence $b_{n+k}^{j}=b_{n+k}^{j^{\prime}}$ and also $\mathbf{b}_{(a, c)}^{j}=\mathbf{b}_{(a, c)}^{j^{\prime}}$. It remains to prove that any admissible sequence $\mathbf{b}_{(a, c)}=$ $\left(b_{l}, b_{l+1}, \ldots\right) \in\{0,1, \ldots, a-1\}^{\mathbb{N}_{l}}$ is equal to some $\mathbf{b}_{(a, c)}^{j}$. It is obvious if $l \leq$ $k$. So, let us assume that $l>k$. Since $p_{i} b_{i+1} \equiv r_{i} b_{i}(\bmod a)$ for $i \geq l$, we claim that $b_{i+1} \equiv x_{i} b_{i}(\bmod a)$ for $i \geq l$. Assume the contrary. Then $p_{i} b_{i+1}$ is not congruent to $p_{i} x_{i} b_{i}(\bmod a)$, which implies that $p_{i} b_{i+1}$ is not congruent to $r_{i} b_{i}(\bmod a)$ and we get a contradiction. Thus $b_{n} \equiv\left(\prod_{i=l}^{n-1} x_{i}\right) b_{l}$ $(\bmod a)$ for $n>l$. If $\left\{d_{i_{1}}, d_{i_{2}}, \ldots, d_{i_{n}}\right\}=\emptyset$, let $j$ be a unique solution of a congruence $\left(\prod_{i=k}^{l-1} x_{i}\right) j \equiv b_{l}(\bmod a)$. Then $b_{l}^{j} \equiv\left(\prod_{i=k}^{l-1} x_{i}\right) j \equiv b_{l}(\bmod a)$ and $\mathbf{b}_{(a, c)}=\mathbf{b}_{(a, c c)}^{j}$. If $\left\{d_{i_{1}}, d_{i_{2}}, \ldots, d_{i_{n}}\right\} \neq \emptyset$, choose large enough $n \geq l>k$ such that $\prod_{i=l}^{n-1} x_{i}=\lambda d_{i_{1}}^{l_{1}} l_{i_{2}}^{l_{2}} \cdots d_{i_{n}}^{l_{n}}$, where $\lambda \in \mathbb{N}, l_{j} \geq \alpha_{i_{j}}$ for $j=1, \ldots, n$ and $d \nmid \lambda$ for each $d \notin\left\{d_{i_{1}}, d_{i_{2}}, \ldots, d_{i_{n}}\right\}$. Then $b_{n} \equiv \lambda d_{i_{1}}^{l_{1}} d_{i_{2}}^{l_{2}} \cdots d_{i_{n}}^{l_{n}} b_{l}(\bmod a)$. Let $j$ be

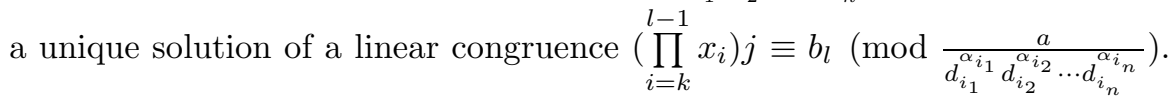
Then $d_{i_{1}}^{\alpha_{i_{1}}} d_{i_{2}}^{\alpha_{i_{2}}} \cdots d_{i_{n}}^{\alpha_{i_{n}}}\left(b_{l}-\left(\prod_{i=k}^{l-1} x_{i}\right) j\right) \equiv 0(\bmod a)$ and we get $b_{n}-b_{n}^{j} \equiv$ $\lambda d_{i_{1}}^{l_{1}} d_{i_{2}}^{l_{2}} \cdots d_{i_{n}}^{l_{n}}\left(b_{l}-b_{l}^{j}\right) \equiv \lambda d_{i_{1}}^{l_{1}} d_{i_{2}}^{l_{2}} \cdots d_{i_{n}}^{l_{n}}\left(b_{l}-\left(\prod_{i=k}^{l-1} x_{i}\right) j\right) \equiv 0(\bmod a)$. This proves $\mathbf{b}_{(a, c)}=\mathbf{b}_{(a, c)}^{j}$. Hence $n_{(a, c)}=\frac{a}{d_{i_{1}}^{\alpha_{i_{1}}} d_{i_{2}}^{\alpha_{i_{2}} \ldots d_{i_{n}}}}$.

Claim 2. If $c$ is odd, then $n_{(a, c)}=a$.

Note that $G C D\left(x_{i}, a\right)=1$ for $i \geq k$ and $b_{k+n}^{j} \equiv\left(\prod_{i=k}^{k+n-1} x_{i}\right) j+$ $\left(\prod_{i=k+1}^{k+n-1} x_{i}\right) y_{k}+\left(\prod_{i=k+2}^{k+n-1} x_{i}\right) y_{k+1}+\cdots+y_{k+n-1} \equiv\left(\prod_{i=k}^{k+n-1} x_{i}\right) j+b_{k+n}^{0}(\bmod a)$ for an arbitrary $n \in \mathbb{N}$. We claim that all sequences $\mathbf{b}_{(a, c)}^{j}, j=0,1, \ldots, a-1$, are different. Assume the contrary. Then there are $j, j^{\prime} \in\{0,1, \ldots, a-1\}, j \neq j^{\prime}$, and $n \in \mathbb{N}$ such that $b_{k+n}^{j}=b_{k+n}^{j^{\prime}}$. Then $\left(\prod_{i=k}^{k+n-1} x_{i}\right)\left(j-j^{\prime}\right) \equiv 0(\bmod a)$. Since $G C D\left(\prod_{i=k}^{k+n-1} x_{i}, a\right)=1$, it follows $j=j^{\prime}$, which is a contradiction. It remains to prove that any admissible sequence $\mathbf{b}_{(a, c)}=\left(b_{l}, b_{l+1}, \ldots\right) \in\{0,1, \ldots, a-1\}^{\mathbb{N}_{l}}$ is equal to some $\mathbf{b}_{(a, c)}^{j}$. It is obvious if $l \leq k$. So, let us assume that $l>k$. 
Since $p_{i} b_{i+1} \equiv b_{i}-q_{i}(\bmod a)$ for $i \geq k$, we claim that $b_{i+1} \equiv x_{i} b_{i}+y_{i}$ $(\bmod a)$ for $i \geq k$. Assume the contrary. Then $p_{i} b_{i+1}$ is not congruent to $p_{i} x_{i} b_{i}+p_{i} y_{i}(\bmod a)$ and consequently $p_{i} b_{i+1}$ is not congruent to $b_{i}-q_{i}$ $(\bmod a)$, which is a contradiction. Let $j$ be a unique solution of a linear congruence $\left(\prod_{i=k}^{l-1} x_{i}\right) j \equiv b_{l}-b_{l}^{0}(\bmod a)$. Then $b_{l}-b_{l}^{j} \equiv b_{l}-\left(\prod_{i=k}^{l-1} x_{i}\right) j-b_{l}^{0} \equiv 0$ $(\bmod a)$, which implies $b_{l}=b_{l}^{j}$. This proves $\mathbf{b}_{(a, c)}=\mathbf{b}_{(a, c)}^{j}$. Hence $n_{(a, c)}=a$.

Note that $n_{(a, c)}$ is even if and only if $a$ is even.

REMARK 6.5. Each admissible sequence $\mathbf{b}_{(a, c)}$ for $\Sigma(\boldsymbol{p}, \boldsymbol{q}, \boldsymbol{r}), c$ even, is a super-admissible sequence for the product $\Sigma(\boldsymbol{p}) \times \Sigma(\boldsymbol{r})$ of solenoids $\Sigma(\boldsymbol{p})$ and $\Sigma(\boldsymbol{r})$ (see [1, Appendix A] and [2]). The proof of Theorem 6.4 related to the case $c$ even is the same as one done for super-admissible sequences in [2].

For $s \in \mathbb{N}$ and sequences $\boldsymbol{p}=\left(\boldsymbol{p}_{i}\right), \boldsymbol{r}=\left(\boldsymbol{r}_{i}\right)$ let $F_{s}$ denote the set of all ordered pairs $(a, c) \in \mathbb{N} \times \mathbb{N}$ satisfying $a c=s$ and $G C D\left(a, p_{i}\right)=G C D\left(c, r_{i}\right)=$ 1 for almost all $i$.

Corollary 6.6. Let $(\Sigma(\boldsymbol{p}, \boldsymbol{q}, \boldsymbol{r}), *)$ be a pointed Klein bottle weak solenoidal space and let $s \in \mathbb{N}$. Then there are $\sum_{(a, c) \in F_{s}} n_{(a, c)}$ different equivalence classes of pointed s-sheeted covering maps over $(\Sigma(\boldsymbol{p}, \boldsymbol{q}, \boldsymbol{r}), *)$ with connected total space.

\section{UNPOINTED FINITE-SHEETED COVERING MAPS OVER $\Sigma(\boldsymbol{p}, \boldsymbol{q}, \boldsymbol{r})$}

Definition 7.1. Let $\mathbf{b}_{(a, c)}, \mathbf{b}_{\left(a^{\prime}, c^{\prime}\right)}^{\prime}$ be admissible sequences for $\Sigma(\boldsymbol{p}, \boldsymbol{q}, \boldsymbol{r})$. We say that $\mathbf{b}_{\left(a^{\prime}, c^{\prime}\right)}^{\prime}$ is conjugate to $\mathbf{b}_{(a, c)}$ provided

(i) $c^{\prime}=c, a^{\prime}=a$;

(ii) $b_{i}^{\prime}=b_{i}$ or $b_{i}^{\prime}=a-b_{i}$ for almost all $i$, if $c$ is even;

(iii) $b_{i}^{\prime}-b_{i}$ is even for almost all $i$ if $c$ is odd and $a$ is even.

Note that conjugacy is an equivalence relation on admissible sequences for $\Sigma(\boldsymbol{p}, \boldsymbol{q}, \boldsymbol{r})$. If both $a$ and $c$ are odd then any two admissible sequence $\mathbf{b}_{(a, c)}, \mathbf{b}_{(a, c)}^{\prime}$ are conjugate.

Proposition 7.2. Let $\mathbf{b}_{(a, c)}$ and $\mathbf{b}_{(a, c)}^{\prime}$ be admissible sequences for $\Sigma(\boldsymbol{p}, \boldsymbol{q}, \boldsymbol{r}) \cdot \mathbf{b}_{(a, c)}$ and $\mathbf{b}_{(a, c)}^{\prime}$ are conjugate if and only if there is a sequence $\left(g_{i}\right) \in G^{\mathbb{N}_{k_{0}}}$ such that $\left\langle\alpha^{a}, \alpha^{b_{i}^{\prime}} \beta^{c}\right\rangle=g_{i}^{-1}\left\langle\alpha^{a}, \alpha^{b_{i}} \beta^{c}\right\rangle g_{i}$ and $h_{\left(p_{i}, q_{i}, r_{i}\right)}\left(g_{i+1}\right) \in$ $\left\langle\alpha^{a}, \alpha^{b_{i}} \beta^{c}\right\rangle g_{i}$ for each $i \geq k_{0} \geq k^{*}$.

Proof. Let $\mathbf{b}_{(a, c)}$ and $\mathbf{b}_{(a, c)}^{\prime}$ be conjugate admissible sequences for $\Sigma(\boldsymbol{p}, \boldsymbol{q}, \boldsymbol{r})$. Then there is $k_{0} \geq k, k^{\prime}$ such that for each $i \geq k_{0}, b_{i}^{\prime}$ is either $b_{i}$ or $a-b_{i}$ if $c$ is even or $b_{i}^{\prime}-b_{i}$ is even if $a$ is even and $c$ is odd. Without loss 
of generality we assume $k_{0}=1$. We will construct the desired sequence $\left(g_{i}\right)$ by the induction.

Let $c$ be even. Since $\mathbf{b}_{(a, c)}$ and $\mathbf{b}_{(a, c)}^{\prime}$ are conjugate, $b_{i}^{\prime}$ is either $b_{i}$ or $a-b_{i}$. The only non-trivial case is $b_{i}^{\prime}=a-b_{i} \neq b_{i}$ for all $i$. Put $g_{1}=\beta$. Then $\beta^{-1}\left\langle\alpha^{a}, \alpha^{b_{1}} \beta^{c}\right\rangle \beta=\left\langle\alpha^{a}, \alpha^{-b_{1}} \beta^{c}\right\rangle=\left\langle\alpha^{a}, \alpha^{a-b_{1}} \beta^{c}\right\rangle$. Since $G C D\left(r_{1}, c\right)=1$ there are integers $v_{2}, k_{2}$ such that $r_{1} v_{2}-c k_{2}=1$. Since $r_{1}$ is odd $v_{2}$ is also odd. Since $G C D\left(p_{1}, a\right)=1$ there are integers $u_{2}$ and $l_{2}$ such that $p_{1} u_{2}-a l_{2}=k_{2} b_{1}-q_{1} v_{2}$. Put $g_{2}=\alpha^{u_{2}} \beta^{v_{2}} \cdot v_{2}$ is odd and therefore $\left(\alpha^{u_{2}} \beta^{v_{2}}\right)^{-1}\left\langle\alpha^{a}, \alpha^{b_{2}} \beta^{c}\right\rangle \alpha^{u_{2}} \beta^{v_{2}}=$ $\left\langle\alpha^{a}, \alpha^{-b_{2}} \beta^{c}\right\rangle=\left\langle\alpha^{a}, \alpha^{a-b_{2}} \beta^{c}\right\rangle$. Furthermore,

$$
\begin{aligned}
h_{\left(p_{1}, q_{1}, r_{1}\right)}\left(g_{2}\right) & =h_{\left(p_{1}, q_{1}, r_{1}\right)}\left(\alpha^{u_{2}} \beta^{v_{2}}\right)=\alpha^{p_{1} u_{2}+q_{1} v_{2}} \beta^{r_{1} v_{2}} \\
& =\alpha^{p_{1} u_{2}+q_{1} v_{2}} \beta^{c k_{2}+1}=\alpha^{p_{1} u_{2}+q_{1} v_{2}-k_{2} b_{1}}\left(a^{b_{1}} \beta^{c}\right)^{k_{2}} \beta \\
& =\alpha^{l_{2} a}\left(\alpha^{b_{1}} \beta^{c}\right)^{k_{2}} \beta \in\left\langle\alpha^{a}, \alpha^{b_{1}} \beta\right\rangle g_{1} .
\end{aligned}
$$

Let us assume that for each $i=1, \ldots, n$ we have constructed $g_{i}=\alpha^{u_{i}} \beta^{v_{i}}$, $v_{i}$ odd, such that $\left\langle\alpha^{a}, \alpha^{a-b_{i}} \beta^{c}\right\rangle=g_{i}^{-1}\left\langle\alpha^{a}, \alpha^{b_{i}} \beta^{c}\right\rangle g_{i}, i=1, \ldots, n$ and $h_{\left(p_{i}, q_{i}, r_{i}\right)}\left(g_{i+1}\right) \in\left\langle\alpha^{a}, \alpha^{b_{i}} \beta^{c}\right\rangle g_{i}, i=1, \ldots, n-1$. Since $G C D\left(r_{n}, c\right)=1$ there are integers $v_{n+1}, k_{n+1}$ such that $r_{n} v_{n+1}-c k_{n+1}=v_{n}$. Since $r_{n}$ and $v_{n}$ are odd, $v_{n+1}$ is also odd. Since $G C D\left(p_{n}, a\right)=1$ there are integers $u_{n+1}$ and $l_{n+1}$ such that $p_{n} u_{n+1}-a l_{n+1}=k_{n+1} b_{n}-q_{n} v_{n+1}+u_{n}$. Put $g_{n+1}=\alpha^{u_{n+1}} \beta^{v_{n+1}}$. $v_{n+1}$ is odd and therefore

$$
\left(\alpha^{u_{n+1}} \beta^{v_{n+1}}\right)^{-1}\left\langle\alpha^{a}, \alpha^{b_{n}} \beta^{c}\right\rangle \alpha^{u_{n+1}} \beta^{v_{n+1}}=\left\langle\alpha^{a}, \alpha^{-b_{n}} \beta^{c}\right\rangle=\left\langle\alpha^{a}, \alpha^{a-b_{n}} \beta^{c}\right\rangle .
$$

Moreover,

$$
\begin{aligned}
h_{\left(p_{n}, q_{n}, r_{n}\right)}\left(g_{n+1}\right) & =h_{\left(p_{n}, q_{n}, r_{n}\right)}\left(\alpha^{u_{n+1}} \beta^{v_{n+1}}\right) \\
& =\alpha^{p_{n} u_{n+1}+q_{n} v_{n+1}} \beta^{r_{n} v_{n+1}}=\alpha^{p_{n} u_{n+1}+q_{n} v_{n+1}} \beta^{c k_{n+1}+v_{n}} \\
& =\alpha^{p_{n} u_{n+1}+q_{n} v_{n+1}-b_{n} k_{n+1}-u_{n}}\left(a^{b_{n}} \beta^{c}\right)^{k_{n+1}} \alpha^{u_{n}} \beta^{v_{n}} \\
& =\alpha^{l_{n+1} a}\left(\alpha^{b_{n}} \beta^{c}\right)^{k_{n+1}} g_{n} \in\left\langle\alpha^{a}, \alpha^{b_{n}} \beta^{c}\right\rangle g_{n} .
\end{aligned}
$$

Let $c$ be odd. Since $\mathbf{b}_{(a, c)}$ and $\mathbf{b}_{(a, c)}^{\prime}$ are admissible for $\Sigma(\boldsymbol{p}, \boldsymbol{q}, \boldsymbol{r}), p_{i} b_{i+1}^{\prime} \equiv$ $b_{i}^{\prime}-q_{i}(\bmod a)$ and $p_{i} b_{i+1} \equiv b_{i}-q_{i}(\bmod a)$. This implies $p_{i}\left(b_{i+1}-b_{i+1}^{\prime}\right) \equiv$ $b_{i}-b_{i}^{\prime}(\bmod a)$. Furthermore, $b_{i}^{\prime}-b_{i}$ is even if $a$ is even. By the induction we will construct a sequence $\left(u_{i}\right)$ of integers such that $2 u_{i} \equiv b_{i}-b_{i}^{\prime}(\bmod a)$ and $p_{i} u_{i+1} \equiv u_{i}(\bmod a)$. Let $u_{1}$ be a solution of an equation $2 u_{1} \equiv b_{1}-b_{1}^{\prime}$ $(\bmod a)$. Assume that $u_{i}, i=1, \ldots, n$, have desired properties. Let $u_{n+1}$ be a unique solution of an equation $p_{n} u_{n+1} \equiv u_{n}(\bmod a)$. Then $p_{n} 2 u_{n+1} \equiv$ $2 u_{n} \equiv b_{n}-b_{n}^{\prime} \equiv p_{n}\left(b_{n+1}-b_{n+1}^{\prime}\right)(\bmod a)$, which implies $2 u_{n+1} \equiv b_{n+1}-b_{n+1}^{\prime}$ $(\bmod a)$. This proves $u_{n+1}$ has both required properties. Put $g_{i}=\alpha^{u_{i}}$ for every $i$. We get $\left(\alpha^{v_{i}}\right)^{-1}\left\langle\alpha^{a}, \alpha^{b_{i}} \beta^{c}\right\rangle \alpha^{v_{i}}=\left\langle\alpha^{a}, \alpha^{b_{i}-2 v_{i}} \beta^{c}\right\rangle=\left\langle\alpha^{a}, \alpha^{b_{i}^{\prime}} \beta^{c}\right\rangle$. Since $p_{i} u_{i+1} \equiv u_{i}(\bmod a)$ for each $i$ there is an integer $l_{i}$ such that $p_{i+1} u_{i+1}-$ $u_{i}=l_{i} a$. Then $h_{\left(p_{i}, q_{i}, r_{i}\right)}\left(g_{i+1}\right)=h_{\left(p_{i}, q_{i}, r_{i}\right)}\left(\alpha^{u_{i+1}}\right)=\alpha^{p_{i} u_{i+1}}=\alpha^{l_{i} a+u_{i}} \in$ $\left\langle\alpha^{a}, \alpha^{b_{i}} \beta^{c}\right\rangle \alpha^{u_{i}}$. 
Conversely, let $\left(g_{i}=\alpha^{u_{i}} \beta^{v_{i}}\right) \in G^{\mathbb{N}_{k_{0}}}$ be a sequence such that $\left\langle\alpha^{a}, \alpha^{b_{i}^{\prime}} \beta^{c}\right\rangle=$ $g_{i}^{-1}\left\langle\alpha^{a}, \alpha^{b_{i}} \beta^{c}\right\rangle g_{i}$ and $h_{\left(p_{i}, q_{i}, r_{i}\right)}\left(g_{i+1}\right) \in\left\langle\alpha^{a}, \alpha^{b_{i}} \beta^{c}\right\rangle g_{i}$ for each $i$. We claim that $\mathbf{b}_{(a, c)}$ and $\mathbf{b}_{(a, c)}^{\prime}$ are conjugate.

First let $c$ be even. By the assumption,

$$
\begin{aligned}
h_{\left(p_{i}, q_{i}, r_{i}\right)}\left(g_{i+1}\right) & =h_{\left(p_{i}, q_{i}, r_{i}\right)}\left(\alpha^{u_{i+1}} \beta^{v_{i+1}}\right) \\
& =\alpha^{p_{i} u_{i+1}}\left(\alpha^{q_{i}} \beta^{r_{i}}\right)^{v_{i+1}} \in\left\langle\alpha^{a}, \alpha^{b_{i}} \beta^{c}\right\rangle \alpha^{u_{i}} \beta^{v_{i}} .
\end{aligned}
$$

Since all $r_{i}$ are odd and $r_{i} v_{i+1}=c k_{i}+v_{i}$ for some integer $k_{i}$ it follows that all $v_{i}$ are odd or all $v_{i}$ are even. On the other hand $\left(\alpha^{u_{i}} \beta^{v_{i}}\right)^{-1} \alpha^{a}\left(\alpha^{u_{i}} \beta^{v_{i}}\right)=$ $\alpha^{(-1)^{v_{i}} a}$ and $\left(\alpha^{u_{i}} \beta^{v_{i}}\right)^{-1} \alpha^{b_{i}} \beta^{c}\left(\alpha^{u_{i}} \beta^{v_{i}}\right)=\alpha^{(-1)^{v_{i}} b_{i}} \beta^{c}$. Thus either

$$
\left\langle\alpha^{a}, \alpha^{b_{i}^{\prime}} \beta^{c}\right\rangle=g_{i}^{-1}\left\langle\alpha^{a}, \alpha^{b_{i}} \beta^{c}\right\rangle g_{i}=\left\langle\alpha^{a}, \alpha^{b_{i}} \beta^{c}\right\rangle
$$

or

$$
\left\langle\alpha^{a}, \alpha^{b_{i}^{\prime}} \beta^{c}\right\rangle=g_{i}^{-1}\left\langle\alpha^{a}, \alpha^{b_{i}} \beta^{c}\right\rangle g_{i}=\left\langle\alpha^{a}, \alpha^{a-b_{i}} \beta^{c}\right\rangle .
$$

Now we conclude that $b_{i}^{\prime}$ is either $b_{i}$ or $a-b_{i}$, which proves that $\mathbf{b}_{(a, c)}$ and $\mathbf{b}_{(a, c)}^{\prime}$ are conjugate.

Let $c$ be odd. First note that

$$
\left(\alpha^{u_{i}} \beta^{v_{i}}\right)^{-1} \alpha^{b_{i}} \beta^{c}\left(\alpha^{u_{i}} \beta^{v_{i}}\right)=\alpha^{(-1)^{v_{i}}\left(b_{i}-2 u_{i}\right)} \beta^{c} \in\left\langle\alpha^{a}, \alpha^{b_{i}^{\prime}} \beta^{c}\right\rangle .
$$

Thus $(-1)^{v_{i}}\left(b_{i}-2 u_{i}\right) \equiv b_{i}^{\prime}(\bmod a)$. This shows that an equation $2 x \equiv b_{i}-b_{i}^{\prime}$ $(\bmod a)$ or $2 x \equiv b_{i}+b_{i}^{\prime}(\bmod a)$ has a solution. If $a$ is even this means $b_{i}-b_{i}^{\prime}$ is even. This proves $\mathbf{b}_{(a, c)}$ and $\mathbf{b}_{(a, c)}^{\prime}$ are conjugate.

THEOREM 7.3. Let $\Sigma(\boldsymbol{p}, \boldsymbol{q}, \boldsymbol{r})$ be a Klein bottle weak solenoidal space and let $s \in \mathbb{N}$. Then there is a bijection $F$ between the set of all conjugacy classes of admissible sequences $\mathbf{b}_{(a, c)}$ for $\Sigma(\boldsymbol{p}, \boldsymbol{q}, \boldsymbol{r})$, where ac $=s$, and the set of all equivalence classes of $s$-sheeted covering maps $f: X \rightarrow \Sigma(\boldsymbol{p}, \boldsymbol{q}, \boldsymbol{r})$ with a connected total space $X$.

Proof. Put $F\left(\left[\mathbf{b}_{(a, c)}\right]\right)=\left[F_{*}\left(\mathbf{b}_{(a, c)}\right)\right]$. First we prove that $F$ is welldefined. Let $\mathbf{b}_{(a, c)}^{\prime}$ be conjugate to $\mathbf{b}_{(a, c)}$. Let $F_{*}\left(\mathbf{b}_{(a, c)}\right)=f_{*}$ and $F_{*}\left(\mathbf{b}_{(a, c)}^{\prime}\right)=f_{*}^{\prime}$. Then $\Phi_{*}\left(f_{*}\right)=\left\{\left\langle\alpha^{a}, \alpha^{b_{i}} \beta^{c}\right\rangle, h_{\left(p_{i}, q_{i}, r_{i}\right)}, i \geq i_{0}\right\}$ and $\Phi_{*}\left(f_{*}^{\prime}\right)=$ $\left\{\left\langle\alpha^{a}, \alpha^{b_{i}^{\prime}} \beta^{c}\right\rangle, h_{\left(p_{i}, q_{i}, r_{i}\right)}, i \geq i_{0}^{\prime}\right\}$. According to Proposition 7.2, there is a sequence $\left(g_{i}\right) \in G^{\mathbb{N}_{k_{0}}}$ such that $\left\langle\alpha^{a}, \alpha^{b_{i}^{\prime}} \beta^{c}\right\rangle=g_{i}^{-1}\left\langle\alpha^{a}, \alpha^{b_{i}} \beta^{c}\right\rangle g_{i}$ and $h_{\left(p_{i}, q_{i}, r_{i}\right)}\left(g_{i+1}\right) \in\left\langle\alpha^{a}, \alpha^{b_{i}} \beta^{c}\right\rangle g_{i}$ for each $i \geq k_{0} \geq i_{0}, i_{0}^{\prime}$. This means that $\Phi_{*}\left(f_{*}\right)$ and $\Phi_{*}\left(f_{*}^{\prime}\right)$ are conjugate subprogroups of index $a c$ of $\underline{\pi_{1}}(\Sigma(\boldsymbol{p}, \boldsymbol{q}, \boldsymbol{r}), *)$. According to [5, Theorems 5 and 7] $f$ and $f^{\prime}$ are equivalent covering maps, which proves that $F$ is well-defined. Let $F\left(\left[\mathbf{b}_{(a, c)}\right]\right)=F\left(\left[\mathbf{b}_{(a, c)}^{\prime}\right]\right)$. Then $F_{*}\left(\mathbf{b}_{(a, c)}\right)=f_{*}$ and $F_{*}\left(\mathbf{b}_{(a, c)}^{\prime}\right)=f_{*}^{\prime}$ are equivalent covering maps. So, $\Phi_{*}\left(f_{*}\right)=\left\{\left\langle\alpha^{a}, \alpha^{b_{i}} \beta^{c}\right\rangle, h_{\left(p_{i}, q_{i}, r_{i}\right)}, i \geq i_{0}\right\}$ and $\Phi_{*}\left(f_{*}^{\prime}\right)=\left\{\left\langle\alpha^{a}, \alpha^{b_{i}^{\prime}} \beta^{c}\right\rangle, h_{\left(p_{i}, q_{i}, r_{i}\right)}\right.$, $\left.i \geq i_{0}^{\prime}\right\}$ are conjugate subprogroups of index ac of $\pi_{1}(\Sigma(\boldsymbol{p}, \boldsymbol{q}, \boldsymbol{r}), *)$, which means that there is a sequence $\left(g_{i}\right) \in G^{\mathbb{N}_{k_{0}}}$ such that $\left\langle\alpha^{a}, \alpha^{b_{i}^{\prime}} \beta^{c}\right\rangle=$ 
$g_{i}^{-1}\left\langle\alpha^{a}, \alpha^{b_{i}} \beta^{c} g_{i}\right\rangle$ and $h_{\left(p_{i}, q_{i}, r_{i}\right)}\left(g_{i+1}\right) \in\left\langle\alpha^{a}, \alpha^{b_{i}} \beta^{c}\right\rangle g_{i}$ for each $i \geq k^{*} \geq i_{0}, i_{0}^{\prime}$. This means that $\Phi_{*}\left(f_{*}\right)$ and $\Phi_{*}\left(f_{*}^{\prime}\right)$ are conjugate subprogroups of index $a c$ of $\underline{\pi_{1}}(\Sigma(\boldsymbol{p}, \boldsymbol{q}, \boldsymbol{r}), *)$. According to Proposition $7.2, \mathbf{b}_{(a, c)}$ and $\mathbf{b}_{(a, c)}^{\prime}$ are conjugate, $\left[\mathbf{b}_{(a, c)}\right]=\left[\mathbf{b}_{(a, c)}^{\prime}\right]$ and $F$ is an injection.

Let $f: X \rightarrow \Sigma(\boldsymbol{p}, \boldsymbol{q}, \boldsymbol{r})$ be an $s$-sheeted covering map with a connected total space $X$. Let $x \in f^{-1}(*) \subseteq X$ be an arbitrary point. According to Theorem 6.2 , there is an admissible sequence $\mathbf{b}_{(a, c)}$ for $\Sigma(\boldsymbol{p}, \boldsymbol{q}, \boldsymbol{r})$ such that $F_{*}\left(\mathbf{b}_{(a, c)}\right)=f_{*}$. Then $F\left(\left[\mathbf{b}_{(a, c)}\right]\right)=\left[F_{*}\left(\mathbf{b}_{(a, c)}\right)\right]=[f]$.

THEOREM 7.4. Let $N_{(a, c)}$ denote the total number of different conjugacy classes of admissible sequences $\mathbf{b}_{(a, c)}$ for $\Sigma(\boldsymbol{p}, \boldsymbol{q}, \boldsymbol{r}),(a, c) \in \mathbb{N} \times \mathbb{N}$ fixed. Then

$$
N_{(a, c)}= \begin{cases}1, & \text { a odd }, \text { c odd } \\ 2, & \text { a even }, \text { c odd } \\ \frac{n_{(a, c)}}{2}+1, & \text { a even, c even } \\ \frac{n_{(a, c)}+1}{2}, & \text { a odd, c even }\end{cases}
$$

Proof. (i) $a$ odd, $c$ odd. Each two admissible sequences $\mathbf{b}_{(a, c)}$ and $\mathbf{b}_{(a, c)}^{\prime}$ for $\Sigma(\boldsymbol{p}, \boldsymbol{q}, \boldsymbol{r})$ are conjugate and $N_{(a, c)}=1$.

(ii) $a$ even, $c$ odd. Let $\mathbf{b}_{(a, c)}$ and $\mathbf{b}_{(a, c)}^{\prime}$ be admissible sequences for $\Sigma(\mathbf{p}, \mathbf{q}, \mathbf{r})$ such that there is $i$ with $b_{i}-b_{i}^{\prime}$ even. Put $b_{i}-b_{i}^{\prime}=2 k$. Then $p_{i} b_{i+1} \equiv b_{i}-q_{i} \equiv b_{i}^{\prime}+2 k-q_{i}(\bmod a), p_{i} b_{i+1}^{\prime} \equiv b^{\prime}-q_{i}(\bmod a)$ and $p_{i}\left(b_{i+1}-b_{i+1}^{\prime}\right) \equiv 2 k(\bmod a)$. Since $a$ is even and $G C D\left(a, p_{i}\right)=1$ it follows $b_{i+1}-b_{i+1}^{\prime}=2 k^{\prime}$. By the induction we show that $b_{j}-b_{j}^{\prime}$ is even for all $j \geq i$, which shows that $\mathbf{b}_{(a, c)}$ and $\mathbf{b}_{(a, c)}^{\prime}$ are conjugate. Let $\mathbf{b}_{(a, c)}$ and $\mathbf{b}_{(a, c)}^{\prime}$ be admissible sequences for $\Sigma(\mathbf{p}, \mathbf{q}, \mathbf{r})$ such that there is $i$ with $b_{i}-b_{i}^{\prime}$ odd. Let $b_{i}-b_{i}^{\prime}=2 k+1$. Then $p_{i}\left(b_{i+1}-b_{i+1}^{\prime}\right) \equiv 2 k+1(\bmod a)$. Since $p_{i}$ is odd, it follows that $b_{i+1}-b_{i+1}^{\prime}$ is odd. By the induction we prove that $b_{j}-b_{j}^{\prime}$ is odd for all $j \geq i$ and $\mathbf{b}_{(a, c)}$ and $\mathbf{b}_{(a, c)}^{\prime}$ are not conjugate. Consider admissible sequences $\mathbf{b}_{(a, c)}^{j}, j=0, \ldots, a-1$, which represent all different admissible sequences $\mathbf{b}_{(a, c)}$ for $\Sigma(\mathbf{p}, \mathbf{q}, \mathbf{r}) . \mathbf{b}_{(a, c)}^{j}$ and $\mathbf{b}_{(a, c)}^{j^{\prime}}$ are conjugate if and only if $j-j^{\prime}$ is even. It is now clear that there are exactly two different conjugacy classes $\left[\mathbf{b}_{(a, c)}^{0}\right]=\left\{\mathbf{b}_{(a, c)}^{0}, \mathbf{b}_{(a, c)}^{2}, \ldots, \mathbf{b}_{(a, c)}^{a-2}\right\}$ and $\left[\mathbf{b}_{(a, c)}^{1}\right]=\left\{\mathbf{b}_{(a, c)}^{1}, \mathbf{b}_{(a, c)}^{3}, \ldots, \mathbf{b}_{(a, c)}^{a-1}\right\}$, i.e., $N_{(a, c)}=2$.

(iii) $c$ even. Consider admissible sequences $\mathbf{b}_{(a, c)}$ and $\mathbf{b}_{(a, c)}^{\prime}$ such that there is $i$ with $b_{i}^{\prime}=a-b_{i}$. Then $p_{i} b_{i+1}^{\prime} \equiv r_{i} b_{i}^{\prime} \equiv r_{i} a-r_{i} b \equiv-r_{i} b(\bmod a)$ and $p_{i}(a-$ $\left.b_{i+1}\right) \equiv-r_{i} b_{i}(\bmod a)$, which implies $b_{i+1}^{\prime}=a-b_{i+1}$. Thus $b_{j}^{\prime}=a-b_{j}$ for each $j \geq i$. Let $n_{(a, c)}$ be the total number of all different admissible sequences $\mathbf{b}_{(a, c)}$ for $\Sigma(\boldsymbol{p}, \boldsymbol{q}, \boldsymbol{r})$. Recall that $\mathbf{b}_{(a, c)}^{j}=\mathbf{b}_{(a, c)}^{j^{\prime}}$ if and only if $j-j^{\prime} \equiv 0\left(\bmod n_{(a, c)}\right)$ and let $\mathbf{b}_{(a, c)}^{j}, j=0, \ldots, n_{(a, c)}-1$, be representatives of all different admissible sequences. Note that $a-j \equiv n_{(a, c)}-j\left(\bmod n_{(a, c)}\right)$. Hence $\mathbf{b}_{(a, c)}^{j}$ and $\mathbf{b}_{(a, c)}^{n_{(a, c)}-j}$ 
are conjugate. If $n_{(a, c)}$ is even there are $\frac{n_{(a, c)}}{2}+1$ conjugacy classes $\left[\mathbf{b}_{(a, c)}^{0}\right]=$ $\left\{\mathbf{b}_{(a, c)}^{0}\right\},\left[\mathbf{b}_{(a, c)}^{1}\right]=\left\{\mathbf{b}_{(a, c)}^{1}, \mathbf{b}_{(a, c)}^{n_{(a, c)}-1}\right\}, \ldots,\left[\mathbf{b}_{(a, c)}^{\frac{n_{(a, c)}}{2}}\right]=\left\{\mathbf{b}_{(a, c)}^{\frac{n_{(a, c)}}{2}}\right\}$. If $n_{(a, c)}$ is odd then there are $\frac{n_{(a, c)}+1}{2}$ conjugacy classes $\left[\mathbf{b}_{(a, c)}^{0}\right]=\left\{\mathbf{b}_{(a, c)}^{0}\right\},\left[\mathbf{b}_{(a, c)}^{1}\right]=$ $\left\{\mathbf{b}_{(a, c)}^{1}, \mathbf{b}_{(a, c)}^{n_{(a, c)}-1}\right\}, \ldots,\left[\mathbf{b}_{(a, c)}^{\frac{n_{(a, c)}-1}{2}}\right]=\left\{\mathbf{b}_{(a, c)}^{\frac{n_{(a, c)}-1}{2}}, \mathbf{b}_{(a, c)}^{\frac{n_{(a, c)}+1}{2}}\right\}$. If $a$ is even, then $n_{(a, c)}$ is even and $N_{(a, c)}=\frac{n_{(a, c)}}{2}+1$. If $a$ is odd, then $n_{(a, c)}$ is odd and $N_{(a, c)}=\frac{n_{(a, c)+1}}{2}$.

Corollary 7.5. Let $\Sigma(\boldsymbol{p}, \boldsymbol{q}, \boldsymbol{r})$ be a Klein bottle weak solenoidal space and let $s \in \mathbb{N}$. Then there are $\sum_{(a, c) \in F_{s}} N_{(a, c)}$ different equivalence classes of s-sheeted covering maps over $\Sigma(\boldsymbol{p}, \boldsymbol{q}, \boldsymbol{r})$ with connected total space.

EXAMPLE 7.6. Let $\Sigma(\boldsymbol{p}, \boldsymbol{q}, \boldsymbol{r})$ be a Klein bottle weak solenoidal space, where $p_{i}=3, r_{i}=5$ for each $i$. First note that $\Sigma(\boldsymbol{p}, \boldsymbol{q}, \boldsymbol{r})$ admits an $s$ sheeted covering map with a connected total space for each $s \in \mathbb{N}$. We will examine 15-sheeted and 20-sheeted covering maps over $\Sigma(\boldsymbol{p}, \boldsymbol{q}, \boldsymbol{r})$. If $s=15$, then $F_{15}=\{(5,3)\}, n_{(5,3)}=5$ and $N_{(5,3)}=1 . \Sigma(\boldsymbol{p}, \boldsymbol{q}, \boldsymbol{r})$ admits 5 different equivalence classes of pointed 15-sheeted covering maps and all total spaces are homeomorphic to the base space. On the other hand there is only one equivalence class of 15 -sheeted covering maps over $\Sigma(\boldsymbol{p}, \boldsymbol{q}, \boldsymbol{r})$. If $s=20$, then $F_{20}=\{(5,4),(10,2),(20,1)\}, n_{(5,4)}=\frac{5}{5}=1, n_{(10,2)}=\frac{10}{5}=2, n_{(20,1)}=20$, $N_{(5,4)}=\frac{1+1}{2}=1, N_{(10,2)}=\frac{2}{2}+1=2, N_{(20,1)}=2 . \Sigma(\boldsymbol{p}, \boldsymbol{q}, \boldsymbol{r})$ admits 23 different equivalence classes of pointed 20-sheeted covering maps. Among them there are 3 equivalence classes with total space homeomorphic to toroidal groups and 20 equivalence classes with total spaces homeomorphic to the base space. On the other hand there are 5 different equivalence classes of 20 -sheeted covering maps over $\Sigma(\boldsymbol{p}, \boldsymbol{q}, \boldsymbol{r}), 3$ with total spaces homeomorphic to toroidal groups and 2 with total spaces homeomorphic to the base space.

\section{REFERENCES}

[1] K. Eda and V. Matijević, Finite sheeted covering maps over 2-dimensional connected, compact abelian group, Topology Appl. 153 (2006), 1033-1045.

[2] K. Eda and V. Matijević, Finite-index supergroups and subgroups of torsionfree Abelian groups of rank two, submitted.

[3] J. Keesling and S. Mardešić, A shape fibration with fibers of different shape, Pacific J. Math. 84 (1979) 319-331.

[4] S. Mardešić and V. Matijević, Classifying overlay structures of topological spaces, Topology Appl. 113 (2001), 167-209.

[5] V. Matijević, Classifying finite-sheeted covering mappings of paracompact spaces, Rev. Mat. Complut. 16 (2003) 311-327.

[6] M. C. McCord, Inverse limit sequences with covering maps, Trans. Amer. Math. Soc. 114 (1965), 197-209.

[7] C. Tezer, Shape classification of Klein-bottle-like continua, Quart. J. Math. Oxford Ser (2) 40 (1989), 225-243. 
V. Matijević

Department of Mathematics

University of Split

N. Tesle 12, 21000 Split

Croatia

E-mail: vlasta@pmfst.hr

Received: 2.10.2006. 